\title{
ASSESSMENT AND MAPPING THE SOIL DEGRADATION USING GIS AND DEGRADATION INDICES IN AL REYAD PROVINCE, KAFR EL- SHEIKH GOVERNORATE, EGYPT
}

\author{
Y. K. El Ghonamey \\ Soils, Water and Environment Research Institute -ARC - Giza \\ E: mail: kotby72@gmail.com
}

Received: Oct. 23,2018

Accepted: Oct. 31,2018

\begin{abstract}
This study aims to assess and mapping the land capability and soil degradation in Reyad province, Kafr El-Sheikh governorate. To achieve this purpose, satellite images were interpreted and handled using GIS technique. Thirty soil profiles in addition to fifty-six minipits were chosen representing soils of the studied area.

The soil units of the studied area were created and mapping based on the spatial variability of soil salinity and soil sodicity. The results indicated that, the largest soil unit with about $56.5 \%$ of the total studied area is "slightly saline, non-sodic soils". The second unit is the "slightly saline, sodic soils" that occupies about $5 \%$ of the studied area. In addition, "moderately saline, non-sodic soils" unit occupies 3.5\% from studied area as small patches. Another "moderately saline, sodic soils" unit covers about 2.3\%. The smallest unit is "highly saline, sodic soils" that covers about $1 \%$ of the total studied area. The variations between soil units are rendered mainly to the using of agricultural drainage water in irrigation.
\end{abstract}

The capability evaluation indicated that, the studied area have two classes. The first class is the "moderately suitable for agriculture" S2 that occupies $67 \%$ of the area and affected with texture as a main limiting factor. The second class is the "marginally suitable" S3 that found in $1 \%$ from the area and suffering from texture, salinity and sodicity as main limiting factors

Studied Chemical Degradation Indices (CDI) vary from very low to high. The largest class includes the moderate degraded soils that covers about $42 \%$ of the studied area. The soils having low CDI covers about $14 \%$. About $11 \%$ of the total studied area have a high CDI. The remaining are fishpond (29.98 \%) and urban areas (2.07\%).

Studied Biological Degradation Indices (BDI) indicated that the largest area (40\%) could be affiliated to the moderate Biological Degradation class that having low organic matter content because of the prevailing semiarid conditions.

Key words: Soil unit, land capability evaluation, suitability for agriculture, chemical \& biological degradation, GIS.

\section{INTRODUCTION}

Different forms of land degradation affect many of the old agricultural soils of Egypt. The chemical soil degradation is one from these forms noticed and revealed in the irrigated soils of the Nile Delta by many soil researchers (Abdel Kawy and Ali 2012, Shalaby et al. 2012, Wahab et al. 2010).
Land degradation is the process of reducing land suitable for agricultural purposes especially in arid, semi-arid and sub-humid areas as a result of human activities and climatic variations (Barbero-Sierra et al., 2015) and eventually puts livelihoods and sustainable development at risk (Fleskens and Stringer, 2014). It is the alteration in ecological and economic functions due to 
the decrease in the productivity and quality of the land (Hill et al., 2005). Soil salinization is a common occurrence in semiarid and arid regions, where evapotranspiration exceeds rainfall, resulting in accumulation of salts in the root zone (Derici, 2002).

Under Mediterranean conditions, soil can lose its potential productivity mainly due to salt accumulation or sodicity. Soils with high soluble salt content or high exchangeable sodium or with a low cation exchange capacity will correspond to the soils with a higher chemical degradation. This could be rendered to natural conditions and/or human activity (De Paz et al. 2006). From this prospective, finding procedure to control land degradation is an urgent need. The first process of this procedure involves in identification and assessment of the land degradation status. The second is establishing a strategy to combat soil degradation. Several methods have been developed to provide a procedure for land degradation assessment. Such methods have been proposed as expert opinion, remote sensing, field monitoring, and productivity measurements as efficient for the assessment of degraded land. There is no single standardized method for assessment of soil degradation (Tetteh, 2015).

Organic matter is the main nutrient source for plants and microorganisms. Biological degradation Index (BDI) is related to the depletion of organic matter content (De Paz et al. 2006).

The objectives for this study are: (a) Evaluate and mapping the land capability of the studied area. (b) Assess and mapping soil degradation status in Reyad province, Kafr El Sheikh governorate.

\section{MATERIALS AND METHODS}

\section{Location}

The studied area is located in Al Reyad province, Kafr El Sheikh governorate located at the north of Nile delta. It is bounded by El-Burlus Lake at the North, Kafr El-Sheikh province at the south, Al Hamol province at the east and Sedi Salem at the west, with an area of about 80377 Feddans (Fig., 1).

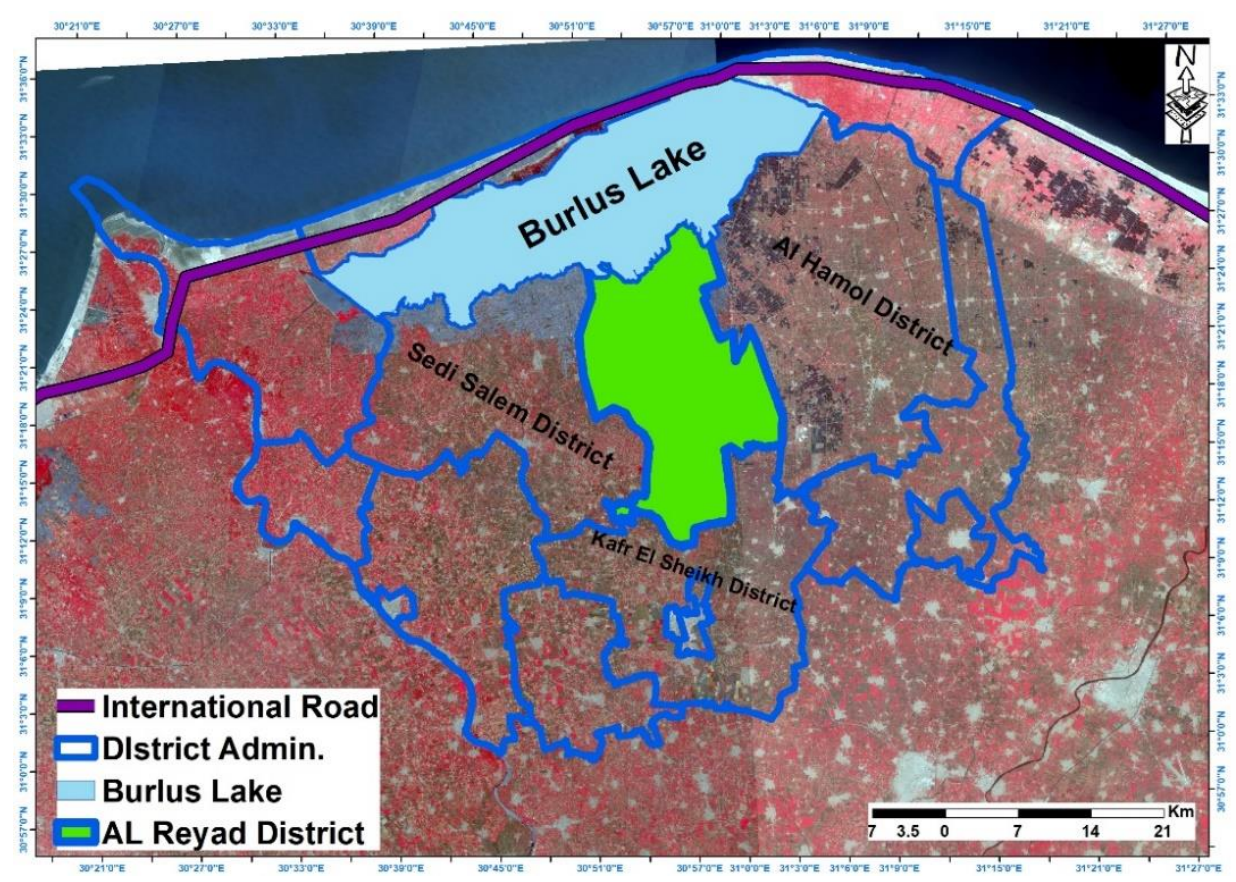

Fig. (1): Location map of the studied area. 


\section{Satellite data:}

Data of Sentinel 2 dated in April 2018 with spatial resolution of $10 \mathrm{~m}$ and spectral resolution of the bands 5,3 and 2 were used for visual interpretation of the studied area (Fig., 2).

Color enhancement operations were used to create new images which is increased the amount of information that can be visually interpreted from the data (Daels, 1986).

Universal Transverse Mercator projection (UTM) was used as a main projection of all data and output maps (Daels, 1986).

The geo-statistical analysis techniques were used to create Digital Elevation
Model (DEM) using the semi-variogram parameters (Stein, 1998) of contour lines and spot heights.

\section{Field Work:}

Thirty soil profiles were selected and 56 minipits were collected (eighty-six soil observation sites) to represent the soils of the studied area. The soil profiles were morphological described according to FAO (2006). Soil samples were collected from different layers of soil profiles in addition to the minipits for laboratory analyses.

Water samples were collected from the 4 main drain canals in the studied area.

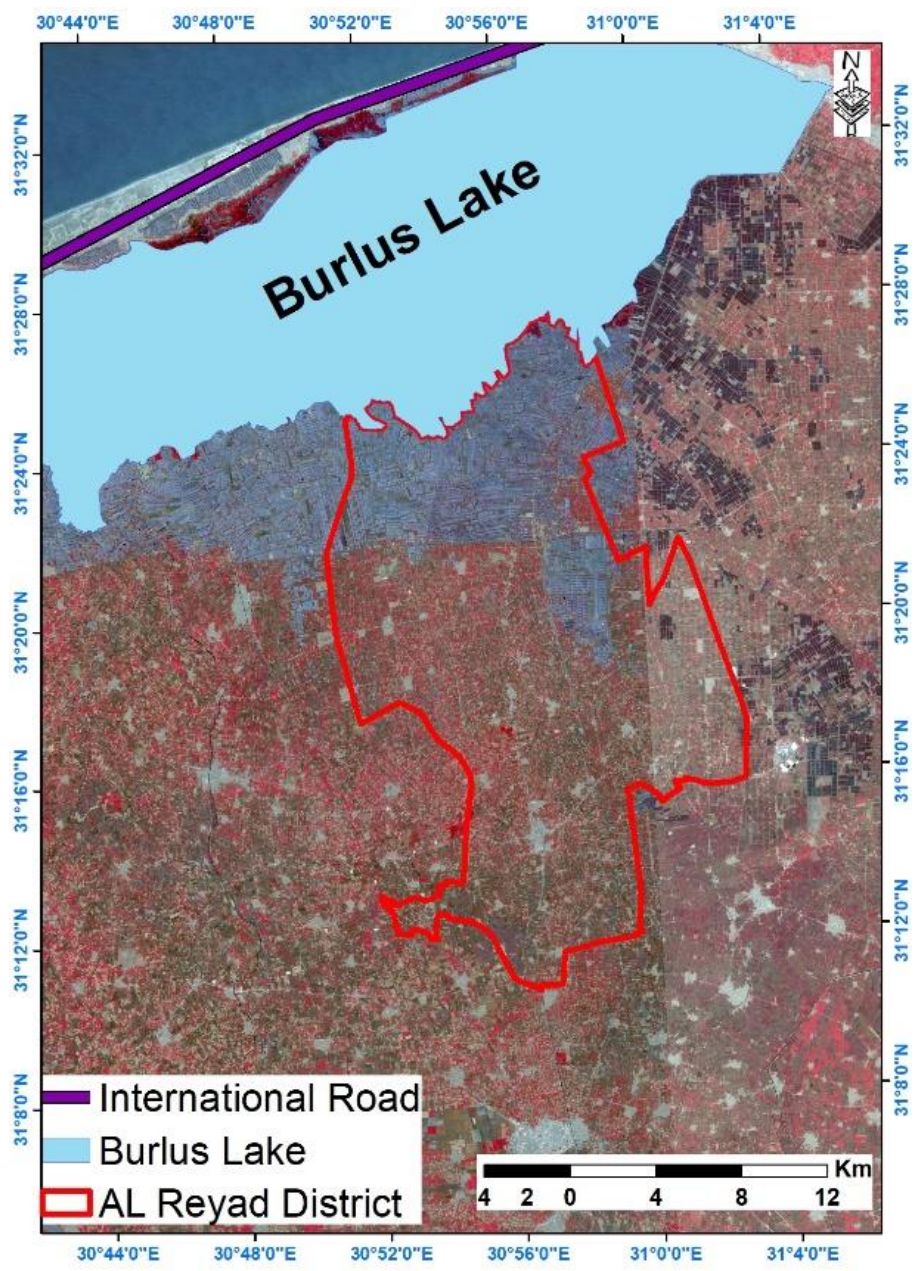

Fig. (2): Sentinel image for the studied area 


\section{Laboratory Analyses:}

The collected soil samples were air dried, crushed and prepared for laboratory analyses, to determine soil chemical and physical properties according to Soil Survey Staff (2004). These properties were particle size distribution, soil $\mathrm{pH}$, electrical conductivity (ECe) in the soil paste extract, cation exchange capacity, exchangeable sodium percentage (ESP) and organic matter content (OM).

Drainage water samples were analyses to determine some chemical properties according to Soil Survey Staff (2004). These included the electric conductivity (ECe), soluble cations and anions and SAR. Suitability of water for irrigation was determine according to the limitations outlined by FAO (1985).

\section{Land Capability Evaluation:}

Land capability was evaluated as suitability for agriculture according to FAO (1985), Sys and Verheye (1978) and Sys et al. (1991). The following equation was used to calculate the capability index Ci:

$$
C i=\frac{t}{100} \times \frac{w}{100} \times \frac{s_{1}}{100} \times \frac{s_{2}}{100} \times \frac{n}{100} \times 100
$$

Where:

$\mathbf{C i}=$ Capability index (\%), $\mathbf{t}=$ Slope, $\mathbf{w}=$ Drainage status, $S_{1}=$ Texture, $S_{2}=$ Soil depth and $\mathbf{n}=$ Salinity and alkalinity

The capability classes were defined according to the values of this index (Sys, 1991) as follows:

\begin{tabular}{|c|c|l|}
\hline $\begin{array}{c}\text { Capability } \\
\text { index (Ci) } \\
\%\end{array}$ & \multicolumn{2}{|c|}{ Capability classes } \\
\hline$>75$ & S1 & $\begin{array}{l}\text { highly suitable for } \\
\text { agriculture. }\end{array}$ \\
\hline $75-50$ & S2 & moderately suitable \\
\hline $50-25$ & S3 & marginally suitable \\
\hline$<25$ & $\mathrm{~N}$ & not suitable \\
\hline
\end{tabular}

6. Soil degradation assessment:
The parameters of geo-statistical approach of the surface layers (eighty-six soil samples) were dealt with Arc GIS 10.3 to produce the maps for distribution of soil salinity, sodicity and organic matter in the studied area. From the semivariogram operation, it could be possible define which models fitted to the experimental semi-variogram values. Parameters of the best fitting model were used to interpolate the thematic soil properties based on ordinary Kriging prediction (Stein, 1998).

The degraded status is better represented by an index for each degradation process. However, these indices should be as simple as possible (De Paz et al. 2006). Chemical and biological soil degradation indices were selected based on the methodology developed by FAO (1980), and applied by Sanchez et al. (1998 and 1999) and De Paz et al. (2006) within the Mediterranean region.

Statistical analysis was done using SPSS program, version 17.0 (2008) was used to test the relation between field capacity and the clay content $\%$, silt content $\%$, sand content, cation exchange capacity, electrical conductivity, exchangeable sodium values of soil samples. The system provides a selection of top quality statistics and a high resolution graphics.

Chemical degradation index (CDI): Chemical degradation index was calculated using the following equations:

$$
\mathrm{CDI}=\frac{\text { Salts }+\mathrm{Na}}{\mathrm{CEC}} \quad \ldots \text { eq. } 1(\mathrm{FAO}, 1980)
$$

Salts $(\mathrm{meq} / \mathbf{1 0 0 g})=\left(13.5 \times \mathrm{EC}_{\mathrm{e}} \mathrm{X}\right.$ Hs $) / 1000$ eq.2 (De Paz et al., 2006)

Hs $=28.215+6.09 \times$ OM + 0.243 X Clay $(\%)-$ 0.11 X Sand (\%)... eq.3 (De Paz et al., 2006) 
Where: Salts is soluble salt content (meq $\left.100 \mathrm{~g}^{-1}\right), \mathrm{Hs}$ is soil water content \% at saturation, $\mathrm{Na}$ is exchangeable sodium (meq $\left.100 \mathrm{~g}^{-1}\right), \quad E C_{e}$ is soil electrical conductivity (dS $\left.\mathrm{m}^{-1}\right), \mathrm{OM}$ is organic matter content (\%), and CEC is cation exchange capacity (meq $100^{-1} \mathrm{~g}$ ).

The multi regression operation was used to determine the formula for the field capacity. Based on the correlation between the soil properties of profiles with the results of field capacity, multi regression formula was created to estimate the field capacity of the minipits. The linear regression model applied on this study assumes that, the $M$ mean of the response variable $Y$ depends on the explanatory variable $X$ according to a linear equation. In the multiple setting, the response variable $Y$ depends on not one but $B$ explanatory variables. The mean response is a linear function of the explanatory variables as following:

$M Y=B_{0}+B_{1} X_{1}+B_{2} X_{2}+\ldots+B p X p$

$M Y$ is the mean estimated field capacity, B0, $B_{1}, B_{2}$ and $B_{p}$ are constants, $X_{1}, X_{2, \ldots .} X_{p}$ are the significant of the relation analysis of soil properties.

Biological degradation index (BDI): Biological degradation is related to the high decomposition rate of organic matter under semiarid conditions. Organic matter $(\mathrm{OM})$ is one of the main nutrient sources for plants and microorganisms. It affects soil aggregation and prevents crusting (De Paz et al. 2006). BDI considers organic matter content alone as the main factor of biological degradation.

$$
\mathrm{BDI}=\frac{1}{\mathrm{OM}} \quad \ldots \ldots \ldots \text { eq. } 4(\mathrm{FAO}-1980)
$$

Description of the chemical and biological degradation degree was indicated according to the rating assigned in Table (1).

\section{RESULTS AND DISCUSSION}

\section{Digital Elevation Model (DEM)}

Fig. (3) shows that, the elevation of the southern studied area ranged from 1.3 to 4.3 meter above sea level. The areas located adjacent to Burlus Lake have low elevation between 0.0 and 0.6 meter above sea level. There are some scattered areas having relatively high elevation areas are found inside the low lands in the north and northwest parts.

\section{Land cover}

The visual interpretation of sentinel satellite image and field check were used to produce land cover map dated in 2018 and presented in Fig. (4) and Table (2).

Table (1): Degree and index ratings of soil chemical and biological degradation (De Paz et al., 2006).

\begin{tabular}{|l|c|c|}
\hline \multicolumn{1}{|c|}{$\begin{array}{c}\text { Degradation } \\
\text { degree }\end{array}$} & $\begin{array}{c}\text { Chemical } \\
\text { degradation } \\
\text { index }\end{array}$ & $\begin{array}{c}\text { Biological } \\
\text { degradation } \\
\text { index }\end{array}$ \\
\hline Very low & $0-0.0081$ & $0-0.3$ \\
\hline Low & $0.0081-0.021$ & $0.3-0.6$ \\
\hline Moderate & $0.021-0.046$ & $0.6-1$ \\
\hline High & $0.046-0.085$ & $1-2.5$ \\
\hline Very high & $>0.085$ & $>2.5$ \\
\hline
\end{tabular}




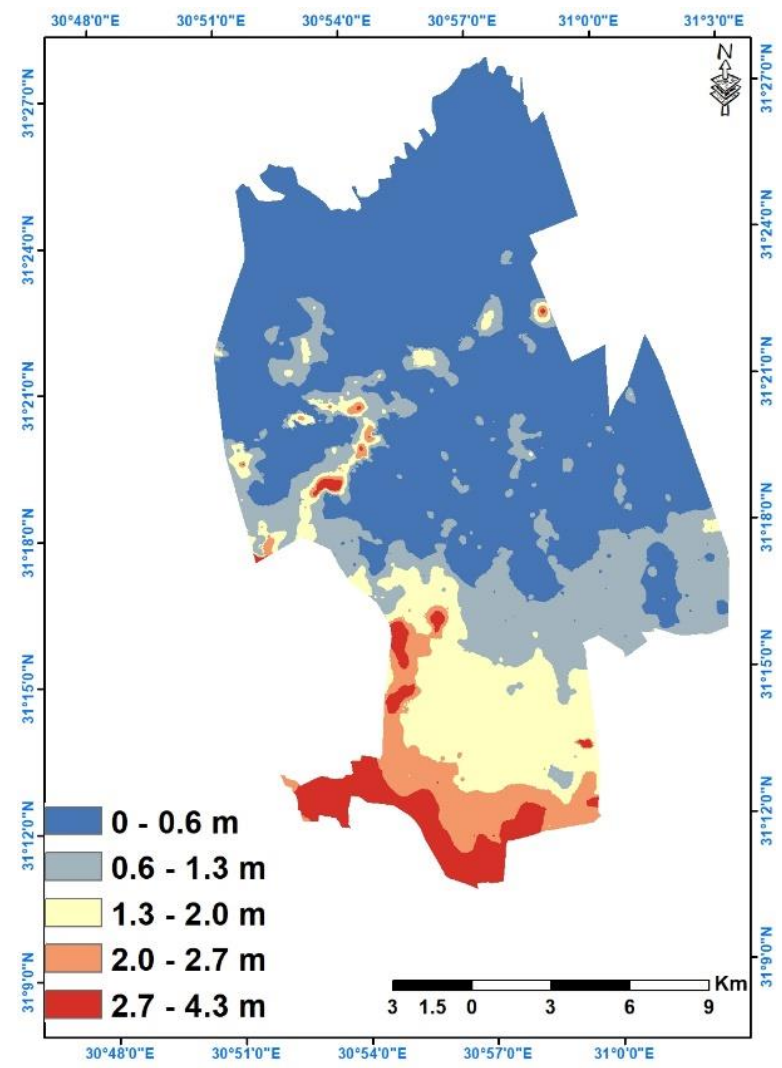

Figure (3). Digital Elevation Model (DEM) of the studied area

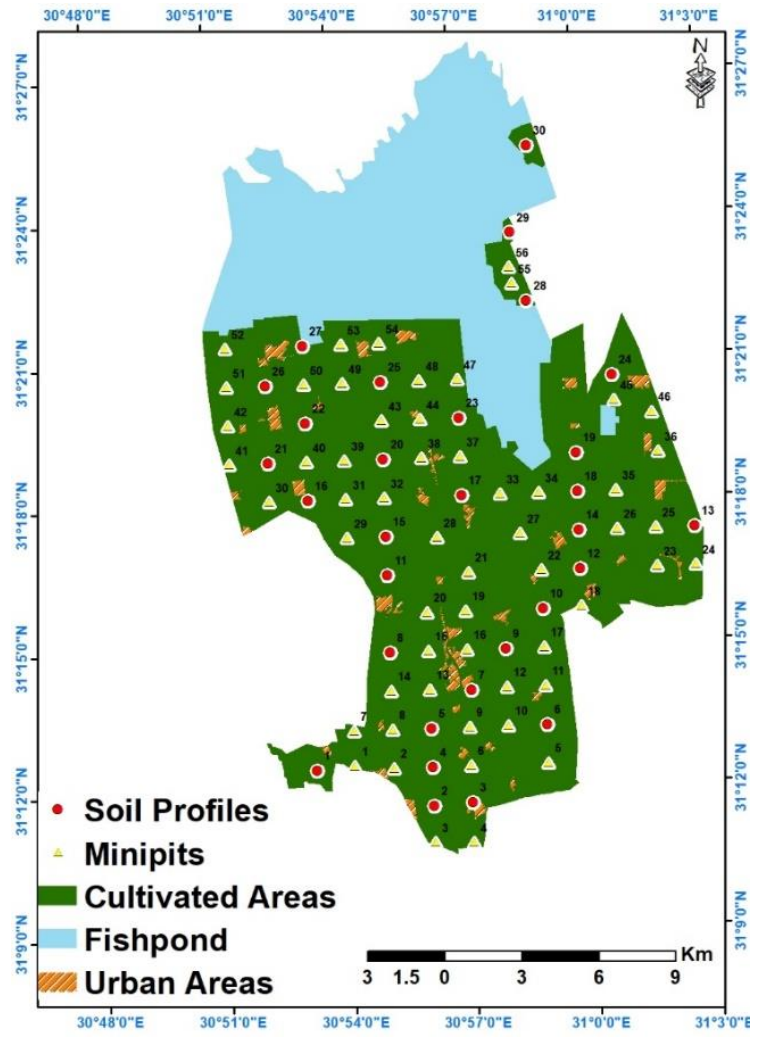

Fig. (4): Land cover map with observation soil points for the studied area 
Assessment and mapping the soil degradation using gis and degradation ..........

Table (2): Areas of the main classes of land cover

\begin{tabular}{|c|c|c|}
\hline Classes & Area in Feddan & $\%$ \\
\hline Cultivated Areas & 54616 & 67.95 \\
\hline Fishpond & 24096 & 29.98 \\
\hline Urban Areas & 1665 & 2.07 \\
\hline Total & 80377 & 100.00 \\
\hline
\end{tabular}

Feddan $=4200 \mathrm{~m}^{2}$

\section{Soil characteristics}

Data in Tables ( 3 and 4$)$ indicated that, these soils are very deep $(>120 \mathrm{~cm}$ in depth). The dominant soil texture is clay as clay content ranges from 41.3 to 67.2 $\%$. The ECe values ranged between 0.2 and $12.6 \mathrm{dS} \mathrm{m}^{-1}$ indicating that, these soils are slightly to highly saline. The soils have alkaline reaction with $\mathrm{pH}$ values between 7.2 and 8.6. Exchangeable sodium percentage ranged from 4 to $27 \%$. CEC ranged between 32.4 to 58.1 meq. $100 \mathrm{~g}^{-1}$. Organic matter content is vary between 0.07 and $3.5 \%$.

\section{Produce thematic maps of the studied area: \\ Soil salinity map}

Soil salinity map was produced using geo-statistical approach for the 86 surface layer samples of the studied area. The salinity classes of the studied soils according to Soil Survey Staff (2004) are given in Table (5) and illustrated as salinity map in Fig. (5). Accordingly, ECe values ranged from 0.2 to $12.62 \mathrm{dS}^{-1}$ with mean of 1.9. and standard deviation of $2.05 \%$. The slightly saline soils cover about $61 \%$ of the total studied area. The moderately and highly saline soils represent an area of about $7 \%$ of the studied area.

\section{Soil sodicity map}

ESP values for the surface layers of the studied area (Tables, 3 and 4 ) are ranged between 0.84 and 29.75 with mean of 9.75 and standard deviation of $6.31 \%$. Accordingly, the sodicity classes of these soils are presented in Table (6) and their map is shown in Fig. (6). Data in Fig. (6) and Table (6) indicated that, the non-sodic soils have ESP $<15 \%$ cover about $60 \%$ of the total studied area. The sodic soils having ESP > 15 cover about $8 \%$ of the total studied area.

\section{Organic matter map}

Organic matter (OM) content of the studied surface soil samples was ranged between $0.53 \%$ and $3.5 \%$ within average of $1.6 \%$ (Tables, 3 and 4 ). The standard deviation is $0.61 \%$. Studied soil classes according their $O M$ contents are presented in Table (7) and illustrated in Fig. (7). The results indicated that the class of soils having low OM contents cover $55.5 \%$ of the total studied area. The soils class having medium OM class cover $12.2 \%$.

\section{Land capability evaluation}

A land capability model for the resulted database was built using Arc GIS 10.3 software to produce the land capability classes and map based on Sys et al., (1991) model (Fig., 8 and Table, 8). Accordingly, the studied soils were classified into two capability classes, i.e. S2 and S3. The soils of S2 class are moderately suitable for agriculture that affected with texture as main limiting factor. This soil class contains area of 53867 Feddans (67\% of the total area). The soils of marginally suitable S3 class occupies an area of 749 Feddans (1\%) that having texture, salinity and sodicity as main limiting factors. 
Table (3): Some chemical and physical properties for soil profiles of the studied area

\begin{tabular}{|c|c|c|c|c|c|c|c|c|c|c|c|c|}
\hline $\begin{array}{c}\text { Prof. } \\
\text { No }\end{array}$ & Depth & pH & $\begin{array}{c}\mathrm{ECe} \\
\mathrm{dSm}-1\end{array}$ & $\begin{array}{c}\text { Sand } \\
\%\end{array}$ & $\begin{array}{l}\text { Silt } \\
\%\end{array}$ & $\begin{array}{c}\text { Clay } \\
\%\end{array}$ & 离 & $\mathrm{FC}_{\%}{ }^{*}$ & $\begin{array}{l}\text { OM } \\
\%\end{array}$ & $\begin{array}{c}\text { Ex Na } \\
\text { meq } \\
100 g^{-1}\end{array}$ & $\begin{array}{c}\text { CEC } \\
\text { meq } \\
100 g^{-1}\end{array}$ & ESP \\
\hline \multirow{4}{*}{1} & $0-25$ & 7.7 & 0.45 & 33.5 & 45.8 & 20.7 & L & 40.0 & 0.97 & 1.5 & 25.9 & 5.8 \\
\hline & $25-50$ & 8.1 & 0.44 & 20.4 & 22.8 & 56.8 & C & 75.4 & 0.90 & 2.7 & 48.4 & 5.6 \\
\hline & $50-125$ & 8.1 & 0.58 & 6.4 & 25.4 & 68.2 & C & 82.1 & 1.10 & 2.3 & 56.4 & 4.1 \\
\hline & $125-150$ & 8.1 & 0.46 & 6.5 & 28.1 & 65.4 & C & 82.1 & 0.58 & 3.5 & 56.4 & 6.1 \\
\hline \multirow[t]{3}{*}{2} & $0-25$ & 7.7 & 0.60 & 13.2 & 34.3 & 52.5 & C & 74.0 & 1.36 & 2.5 & 46.5 & 5.4 \\
\hline & $25-75$ & 7.3 & 0.41 & 13.3 & 34.6 & 53.1 & C & 74.0 & 0.75 & 2.0 & 46.5 & 4.4 \\
\hline & $75-150$ & 7.6 & 0.51 & 13.7 & 34.7 & 51.6 & C & 74.0 & 0.61 & 3.2 & 45.1 & 7.1 \\
\hline \multirow[t]{3}{*}{3} & $0-25$ & 7.7 & 0.52 & 8.8 & 36.9 & 54.3 & C & 74.0 & 1.40 & 3.2 & 49.4 & 6.4 \\
\hline & $25-75$ & 7.6 & 0.68 & 24.6 & 33.8 & 41.6 & C & 66 & 0.85 & 3.4 & 36.8 & 9.2 \\
\hline & $75-130$ & 7.6 & 0.90 & 21.9 & 36.6 & 41.5 & C & 66 & 0.75 & 5.0 & 36.8 & 13.6 \\
\hline \multirow[t]{3}{*}{4} & $0-25$ & 7.6 & 1.65 & 24.3 & 34.4 & 41.3 & C & 67.0 & 1.36 & 3.8 & 35.9 & 10.6 \\
\hline & $25-75$ & 7.4 & 1.15 & 19.5 & 37.7 & 42.8 & C & 66 & 0.75 & 3.4 & 35.9 & 9.6 \\
\hline & $75-150$ & 7.4 & 1.13 & 25.9 & 32.6 & 41.5 & C & 66 & 0.48 & 3.6 & 35.9 & 9.9 \\
\hline \multirow[t]{3}{*}{5} & $0-25$ & 7.9 & 1.06 & 14.4 & 22.3 & 63.3 & C & 81.0 & 1.96 & 2.6 & 55.8 & 4.6 \\
\hline & $25-75$ & 8.2 & 0.32 & 17.5 & 23.0 & 59.5 & C & 80.0 & 0.78 & 1.0 & 51.1 & 2.0 \\
\hline & $75-120$ & 8.2 & 0.33 & 17.5 & 25.0 & 57.5 & C & 78.0 & 0.42 & 0.8 & 50.9 & 1.5 \\
\hline \multirow[t]{3}{*}{6} & $0-20$ & 7.8 & 0.55 & 24.3 & 34.8 & 40.9 & C & 67.0 & 0.98 & 2.2 & 36.7 & 6.1 \\
\hline & $20-70$ & 7.5 & 0.63 & 22.5 & 35.9 & 41.6 & C & 67.5 & 0.58 & 2.8 & 36.7 & 7.5 \\
\hline & $70-130$ & 7.7 & 1.13 & 4,50 & 37.8 & 57.7 & C & 77.3 & 0.31 & 6.7 & 51.2 & 13.2 \\
\hline \multirow[t]{3}{*}{7} & $0-20$ & 7.2 & 0.53 & 16.8 & 39.0 & 44.2 & C & 69.0 & 0.62 & 1.0 & 39.9 & 2.5 \\
\hline & $20-70$ & 7.2 & 0.58 & 16.3 & 38.2 & 45.5 & C & 70.0 & 0.91 & 0.9 & 39.9 & 2.2 \\
\hline & $70-120$ & 8.0 & 0.59 & 14.5 & 39.3 & 46.2 & C & 70.0 & 0.53 & 0.9 & 39.9 & 2.2 \\
\hline \multirow[t]{3}{*}{8} & $0-25$ & 7.8 & 3.75 & 14.3 & 25.4 & 60.3 & C & 80.0 & 1.87 & 4.1 & 52.3 & 7.8 \\
\hline & $25-75$ & 8.0 & 0.50 & 14.0 & 23.5 & 62.5 & C & 80.3 & 0.79 & 1.7 & 53.1 & 3.2 \\
\hline & $75-120$ & 8.1 & 0.89 & 17.3 & 25.4 & 57.3 & C & 79.0 & 0.62 & 2.2 & 50.3 & 4.4 \\
\hline \multirow[t]{3}{*}{9} & $0-20$ & 7.9 & 0.34 & 13.2 & 39.1 & 47.7 & C & 72.0 & 0.85 & 0.8 & 41.3 & 2.0 \\
\hline & $20-70$ & 7.9 & 0.39 & 16.2 & 38.3 & 45.5 & C & 70.0 & 0.79 & 1.0 & 40.6 & 2.5 \\
\hline & $70-120$ & 7.9 & 0.65 & 18.3 & 37.0 & 44.7 & C & 69.0 & 0.59 & 1.2 & 40.6 & 3.0 \\
\hline \multirow{4}{*}{10} & $0-30$ & 7.7 & 1.79 & 13.3 & 34.2 & 51.7 & C & 74.0 & 2.13 & 6.0 & 46.7 & 12.8 \\
\hline & $30-60$ & 7.8 & 2.43 & 11.5 & 33.7 & 54.8 & C & 69.0 & 0.87 & 8.8 & 47.9 & 18.5 \\
\hline & $60-90$ & 7.7 & 3.46 & 11.6 & 35.8 & 52.6 & C & 69.0 & 0.85 & 12.1 & 46.7 & 25.9 \\
\hline & $90-150$ & 7.8 & 1.20 & 6.5 & 36.3 & 57.2 & C & 69.0 & 0.35 & 2.9 & 50.1 & 5.7 \\
\hline
\end{tabular}

$\mathrm{L}=$ Loam, $\mathrm{CL}=$ Clay Loam and C = Clay $\quad \mathrm{FC}^{*}=$ Lap. Field Capacity 
Table (3): Cont.

\begin{tabular}{|c|c|c|c|c|c|c|c|c|c|c|c|c|}
\hline $\begin{array}{l}\text { Prof. } \\
\text { No }\end{array}$ & Depth & $\mathrm{pH}$ & $\begin{array}{c}\text { ECe } \\
\text { dSm-1 }\end{array}$ & $\begin{array}{c}\text { Sand } \\
\%\end{array}$ & $\begin{array}{c}\text { Silt } \\
\%\end{array}$ & $\begin{array}{c}\text { Clay } \\
\%\end{array}$ & 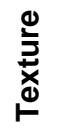 & $\begin{array}{l}\mathrm{FC}_{\%}^{*} \\
\end{array}$ & $\begin{array}{c}\text { OM } \\
\%\end{array}$ & $\begin{array}{c}\text { Ex Na } \\
\text { meq } \\
100 g^{-1}\end{array}$ & $\begin{array}{c}\text { CEC } \\
\text { meq } \\
100 \mathrm{~g}^{-1}\end{array}$ & ESP \\
\hline \multirow{3}{*}{11} & $0-30$ & 7.7 & 1.06 & 11.2 & 22.2 & 54.9 & C & 69.0 & 2.65 & 4.7 & 47.9 & 9.9 \\
\hline & $30-70$ & 7.6 & 0.67 & 11.5 & 11.5 & 54.8 & C & 74.0 & 0.86 & 2.8 & 47.9 & 5.8 \\
\hline & $70-150$ & 7.4 & 0.58 & 11.6 & 11.6 & 52.6 & C & 73.0 & 0.65 & 2.3 & 45.9 & 5.1 \\
\hline \multirow{3}{*}{12} & $0-35$ & 7.7 & 1.27 & 9.4 & 34.7 & 55.9 & C & 76.0 & 2.46 & 2.7 & 50.1 & 5.3 \\
\hline & $35-75$ & 7.7 & 1.18 & 9.9 & 34.9 & 55.4 & C & 77.0 & 0.19 & 4.9 & 50.1 & 9.7 \\
\hline & $75-150$ & 7.7 & 1.43 & 9.5 & 35.7 & 54.8 & C & 75.0 & 0.61 & 7.0 & 50.1 & 14.0 \\
\hline \multirow[t]{3}{*}{13} & $0-30$ & 8.7 & 2.10 & 11.4 & 33.7 & 54.9 & C & 75.0 & 1.99 & 9.8 & 50.9 & 19.3 \\
\hline & $30-85$ & 8.4 & 1.04 & 26.5 & 34.7 & 38.8 & C & 64.0 & 0.95 & 4.4 & 33.1 & 13.2 \\
\hline & $85-130$ & 8.4 & 1.04 & 12.5 & 33.6 & 53.9 & C & 74.0 & 1.74 & 6.7 & 50.9 & 13.2 \\
\hline \multirow[t]{3}{*}{14} & $0-30$ & 7.6 & 1.40 & 9.7 & 35.8 & 54.5 & C & 70.1 & 1.92 & 4.7 & 50.1 & 9.4 \\
\hline & $30-70$ & 7.6 & 2.04 & 19.5 & 36.6 & 43.9 & C & 66.2 & 1.10 & 4.1 & 36.7 & 11.1 \\
\hline & $70-150$ & 7.6 & 1.92 & 9.5 & 35.8 & 54.7 & C & 75.0 & 0.53 & 5.0 & 49.1 & 10.2 \\
\hline \multirow[t]{3}{*}{15} & $0-25$ & 7.7 & 1.26 & 11.5 & 11.5 & 52.8 & C & 74.2 & 1.41 & 3.7 & 44.7 & 8.2 \\
\hline & $25-50$ & 7.4 & 0.89 & 11.6 & 11.6 & 54.7 & C & 75.0 & 0.41 & 3.1 & 48.5 & 6.4 \\
\hline & $50-100$ & 7.6 & 0.90 & 19.4 & 19.4 & 42.8 & C & 66.1 & 0.31 & 2.5 & 37.1 & 6.7 \\
\hline \multirow[t]{3}{*}{16} & $0-30$ & 7.8 & 2.78 & 4.3 & 37.9 & 57.8 & C & 77.0 & 1.32 & 14.1 & 50.3 & 28.0 \\
\hline & $30-75$ & 7.8 & 3.38 & 4.7 & 37.9 & 57.4 & C & 77.1 & 1.18 & 11.2 & 50.3 & 22.2 \\
\hline & $75-150$ & 7.5 & 3.69 & 13.2 & 34.3 & 52.5 & C & 74.1 & 0.77 & 12.1 & 47.6 & 25.5 \\
\hline \multirow[t]{3}{*}{17} & $0-30$ & 7.8 & 0.69 & 13.2 & 34.3 & 52.5 & C & 75.2 & 1.68 & 2.5 & 47.1 & 5.3 \\
\hline & $30-80$ & 7.8 & 0.81 & 13.6 & 34.7 & 51.7 & C & 74.0 & 1.50 & 3.5 & 47.1 & 7.4 \\
\hline & $80-150$ & 7.9 & 0.81 & 21.9 & 36.6 & 41.5 & C & 65.0 & 0.98 & 4.2 & 36.9 & 11.5 \\
\hline \multirow[t]{3}{*}{18} & $0-30$ & 7.7 & 2.48 & 19.3 & 36.8 & 43.9 & C & 67.0 & 1.40 & 5.7 & 36.3 & 15.8 \\
\hline & $30-80$ & 7.7 & 3.24 & 23.3 & 33.4 & 43.3 & C & 67.1 & 0.91 & 6.4 & 36.3 & 17.5 \\
\hline & $80-150$ & 7.7 & 3.58 & 25.3 & 33.8 & 41.9 & C & 66.9 & 0.77 & 6.7 & 36.3 & 18.4 \\
\hline \multirow[t]{3}{*}{19} & $0-30$ & 7.5 & 1.60 & 25.8 & 33.9 & 41.3 & C & 66.1 & 1.56 & 3.5 & 37.1 & 9.5 \\
\hline & $30-60$ & 7.6 & 3.39 & 22.9 & 33.8 & 41.3 & C & 66.3 & 1.01 & 4.2 & 37.1 & 11.2 \\
\hline & $60-100$ & 7.8 & 1.97 & 19.3 & 33.8 & 43.9 & C & 67.1 & 0.59 & 3.8 & 37.1 & 10.1 \\
\hline \multirow[t]{4}{*}{20} & $0-30$ & 7.8 & 1.54 & 4.4 & 36.9 & 58.7 & C & 79.1 & 1.58 & 6.6 & 51.1 & 12.9 \\
\hline & $30-60$ & 7.6 & 1.60 & 23.7 & 33.7 & 42.6 & C & 67.1 & 0.63 & 5.9 & 37.3 & 15.8 \\
\hline & $60-90$ & 7.7 & 1.36 & 25.3 & 33.8 & 40.9 & C & 65.1 & 0.80 & 6.1 & 37.3 & 16.4 \\
\hline & $90-150$ & 7.9 & 1.36 & 24.9 & 33.6 & 41.5 & C & 65.1 & 0.21 & 6.1 & 37.3 & 16.4 \\
\hline \multirow[t]{3}{*}{21} & $0-30$ & 7.5 & 1.20 & 4.7 & 34.7 & 60.6 & C & 80.1 & 1.59 & 5.8 & 51.5 & 11.3 \\
\hline & $30-80$ & 7.4 & 2.40 & 3.7 & 34.8 & 61.8 & $\mathrm{C}$ & 80.1 & 1.02 & 2.0 & 51.5 & 3.8 \\
\hline & $80-130$ & 7.7 & 3.00 & 1.5 & 36.7 & 61.8 & C & 80.1 & 0.95 & 5.0 & 51.5 & 9.8 \\
\hline
\end{tabular}


Table (3): Cont.

\begin{tabular}{|c|c|c|c|c|c|c|c|c|c|c|c|c|}
\hline $\begin{array}{c}\text { Prof. } \\
\text { No }\end{array}$ & Depth & $\mathrm{pH}$ & $\begin{array}{c}\text { ECe } \\
\text { dSm-1 }\end{array}$ & $\begin{array}{c}\text { Sand } \\
\%\end{array}$ & $\begin{array}{c}\text { Silt } \\
\%\end{array}$ & $\begin{array}{c}\text { Clay } \\
\%\end{array}$ & 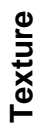 & $\begin{array}{l}\mathbf{F C}_{\%}^{*} \\
\end{array}$ & $\begin{array}{l}\text { OM } \\
\%\end{array}$ & $\begin{array}{l}\text { Ex Na } \\
\text { meq } \\
100 g^{-1}\end{array}$ & $\begin{array}{c}\text { CEC } \\
\text { meq } \\
100 \mathrm{~g}^{-1}\end{array}$ & ESP \\
\hline \multirow{4}{*}{22} & $0-25$ & 7.5 & 1.03 & 12.6 & 34.6 & 52.8 & C & 75.2 & 1.14 & 4.4 & 46.1 & 9.5 \\
\hline & $25-55$ & 7.7 & 4.48 & 19.5 & 37.9 & 42.6 & C & 66.2 & 0.83 & 0.6 & 37.2 & 1.6 \\
\hline & $55-90$ & 7.5 & 3.84 & 20.5 & 37.6 & 41.9 & C & 66.2 & 0.57 & 0.4 & 37.2 & 1.1 \\
\hline & $90-120$ & 7.6 & 3.95 & 19.3 & 37.9 & 42.4 & C & 66.2 & 0.47 & 0.6 & 37.9 & 1.5 \\
\hline \multirow[t]{3}{*}{23} & $0-35$ & 7.7 & 1.23 & 20.5 & 37.6 & 41.9 & C & 65.1 & 1.08 & 2.9 & 35.6 & 8.1 \\
\hline & $35-75$ & 7.7 & 1.76 & 20.4 & 37.8 & 41.8 & C & 65.1 & 1.01 & 3.3 & 35.6 & 9.3 \\
\hline & $75-150$ & 7.9 & 1.76 & 4.4 & 36.9 & 58.7 & C & 79.1 & 0.77 & 4.5 & 50.9 & 8.9 \\
\hline \multirow{3}{*}{24} & $0-25$ & 8.6 & 11.86 & 12.5 & 34.6 & 52.9 & C & 74.2 & 1.43 & 9.7 & 46.9 & 20.7 \\
\hline & $25-70$ & 8.6 & 11.93 & 22.8 & 34.6 & 42.6 & C & 66.1 & 1.32 & 7.7 & 37.7 & 20.5 \\
\hline & $70-130$ & 8.4 & 11.56 & 11.4 & 33.9 & 54.7 & C & 75.0 & 1.27 & 11.8 & 48.1 & 24.6 \\
\hline \multirow{4}{*}{25} & $0-30$ & 7.4 & 2.65 & 4.4 & 36.9 & 58.7 & C & 79.2 & 0.80 & 9.0 & 51.4 & 17.5 \\
\hline & $30-60$ & 7.7 & 5.16 & 6.6 & 36.6 & 56.8 & C & 76.5 & 0.56 & 10.2 & 50.6 & 20.2 \\
\hline & $60-90$ & 7.6 & 3.75 & 12.5 & 33.8 & 53.7 & C & 74.0 & 0.28 & 25.1 & 49.7 & 50.6 \\
\hline & $90-150$ & 8.2 & 5.47 & 23.6 & 33.7 & 42.7 & C & 66.5 & 0.07 & 19.2 & 38.9 & 49.3 \\
\hline \multirow{3}{*}{26} & $0-25$ & 7.9 & 0.76 & 4.8 & 34.7 & 60.5 & C & 80.1 & 2.27 & 3.7 & 51.7 & 7.1 \\
\hline & $25-70$ & 7.9 & 1.01 & 3.4 & 33.9 & 61.8 & C & 80.1 & 0.75 & 6.1 & 51.7 & 11.9 \\
\hline & $70-120$ & 8.5 & 0.86 & 1.9 & 36.8 & 61.3 & C & 80.1 & 1.02 & 3.9 & 51.3 & 7.6 \\
\hline \multirow{4}{*}{27} & $0-30$ & 7.5 & 1.76 & 4.5 & 37.7 & 57.8 & C & 77.3 & 1.58 & 3.4 & 51.1 & 6.7 \\
\hline & $30-60$ & 7.5 & 2.66 & 6.5 & 37.6 & 55.9 & C & 75.1 & 1.10 & 7.4 & 51.0 & 14.5 \\
\hline & $60-90$ & 7.5 & 3.02 & 7.5 & 37.7 & 54.8 & C & 75.1 & 0.93 & 4.8 & 47.9 & 10.0 \\
\hline & $90-150$ & 7.4 & 2.82 & 12.5 & 34.8 & 52.7 & C & 74.0 & 0.38 & 7.4 & 48.1 & 15.5 \\
\hline \multirow{4}{*}{28} & $0-30$ & 7.7 & 2.94 & 23.2 & 33.4 & 43.4 & C & 67.0 & 2.98 & 11.0 & 36.9 & 29.8 \\
\hline & $30-60$ & 7.7 & 5.85 & 11.5 & 34.7 & 53.8 & C & 74.0 & 2.05 & 23.2 & 48.3 & 48.0 \\
\hline & $60-110$ & 7.9 & 2.17 & 12.5 & 34.6 & 52.9 & C & 74.0 & 1.82 & 4.1 & 48.3 & 8.4 \\
\hline & $110-150$ & 7.6 & 2.63 & 11.5 & 33.7 & 54.8 & C & 75.0 & 0.87 & 9.5 & 48.3 & 19.7 \\
\hline \multirow{3}{*}{29} & $0-30$ & 8.6 & 2.70 & 24.4 & 32.9 & 42.7 & C & 67.0 & 1.81 & 4.6 & 36.9 & 12.5 \\
\hline & $30-60$ & 8.5 & 2.80 & 23.5 & 33.6 & 42.9 & C & 67.0 & 1.62 & 6.3 & 36.9 & 17.1 \\
\hline & $60-100$ & 8.4 & 2.19 & 3.5 & 33.8 & 62.7 & C & 81.0 & 0.95 & 9.8 & 53.9 & 18.2 \\
\hline \multirow[t]{3}{*}{30} & $0-25$ & 8.2 & 1.93 & 6.3 & 33.8 & 59.9 & C & 80.1 & 1.12 & 10.1 & 52.5 & 19.3 \\
\hline & $25-70$ & 8.2 & 10.54 & 4.6 & 33.9 & 61.5 & C & 80.1 & 1.10 & 10.2 & 53.7 & 19.1 \\
\hline & $70-130$ & 8.6 & 11.81 & 19.3 & 33.9 & 43.8 & C & 66.9 & 0.96 & 10.9 & 40.1 & 27.2 \\
\hline
\end{tabular}


Assessment and mapping the soil degradation using gis and degradation ..........

Table (4): Some chemical and physical properties for minipits in the studied area

\begin{tabular}{|c|c|c|c|c|c|c|c|c|c|c|c|c|}
\hline $\begin{array}{c}\text { Min. } \\
\text { No }\end{array}$ & Depth & $\mathrm{pH}$ & $\begin{array}{c}\text { ECe } \\
\text { dSm-1 }\end{array}$ & $\begin{array}{c}\text { Sand } \\
\%\end{array}$ & $\begin{array}{l}\text { Silt } \\
\%\end{array}$ & $\begin{array}{c}\text { Clay } \\
\%\end{array}$ & 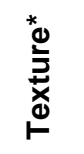 & $\begin{array}{c}\mathbf{F C}^{\star \star} \\
\%\end{array}$ & OM \% & $\begin{array}{c}\text { Ex } \\
\mathrm{Na} \\
\mathrm{meq}^{-100 \mathbf{g}^{-1}}\end{array}$ & $\begin{array}{c}\text { CEC } \\
\text { meq } \\
100 g^{-1}\end{array}$ & ESP \\
\hline 1 & $0-30$ & 8.3 & 0.66 & 17.6 & 25.4 & 55.8 & C & 75.1 & 1.36 & 1.8 & 50.8 & 3.6 \\
\hline 2 & $0-30$ & 8.2 & 0.74 & 24.6 & 35.4 & 33.0 & C L & 55 & 1.24 & 2.1 & 24.1 & 8.7 \\
\hline 3 & $0-30$ & 7.8 & 0.40 & 13.6 & 34.8 & 52.6 & C & 74 & 1.16 & 2.1 & 47.3 & 4.5 \\
\hline 4 & $0-30$ & 7.5 & 0.64 & 18.8 & 37.5 & 43.7 & C & 68 & 1.77 & 2.4 & 36.1 & 6.7 \\
\hline 5 & $0-30$ & 7.8 & 0.57 & 24.4 & 33.7 & 41.9 & C & 67 & 0.98 & 0.7 & 35.7 & 2.1 \\
\hline 6 & $0-30$ & 7.8 & 1.09 & 21.3 & 36.8 & 41.9 & C & 67 & 2.90 & 4.0 & 36.8 & 10.9 \\
\hline 7 & $0-30$ & 8.2 & 0.31 & 11.0 & 21.8 & 67.2 & C & 82 & 1.88 & 0.5 & 55.1 & 0.8 \\
\hline 8 & $0-30$ & 8.0 & 0.20 & 14.5 & 25.0 & 60.5 & C & 80 & 1.92 & 0.5 & 55.1 & 0.8 \\
\hline 9 & $0-30$ & 7.8 & 0.77 & 23.6 & 34.8 & 41.6 & C & 66 & 0.95 & 3.5 & 36.7 & 9.5 \\
\hline 10 & $0-30$ & 7.3 & 0.92 & 11.5 & 33.7 & 54.8 & C & 75 & 1.53 & 4.6 & 49.1 & 9.4 \\
\hline 11 & $0-30$ & 7.9 & 0.61 & 4.8 & 37.7 & 57.5 & C & 78 & 0.75 & 2.8 & 51.6 & 5.5 \\
\hline 12 & $0-30$ & 8.0 & 0.41 & 18.7 & 39.8 & 41.5 & C & 66 & 1.30 & 1.1 & 35.9 & 3.1 \\
\hline 13 & $0-30$ & 8.1 & 1.53 & 19.5 & 20.5 & 60.0 & C & 80 & 2.11 & 3.5 & 51.3 & 6.8 \\
\hline 14 & $0-30$ & 8.3 & 12.62 & 11.5 & 23.2 & 65.3 & C & 85 & 2.02 & 15.9 & 58.1 & 27.3 \\
\hline 15 & $0-30$ & 7.7 & 2.18 & 18.5 & 26.0 & 55.5 & C & 75 & 1.96 & 3.4 & 49.9 & 6.8 \\
\hline 16 & $0-30$ & 8.0 & 0.48 & 13.9 & 38.6 & 47.5 & C & 71 & 0.53 & 0.9 & 32.9 & 2.7 \\
\hline 17 & $0-30$ & 8.0 & 2.64 & 18.8 & 40.7 & 40.5 & Si C & 65 & 0.62 & 1.3 & 31.7 & 4.0 \\
\hline 18 & $0-30$ & 7.9 & 1.91 & 11.7 & 35.6 & 52.7 & C & 67 & 2.02 & 7.1 & 46.7 & 15.3 \\
\hline 19 & $0-30$ & 8.1 & 1.09 & 7.8 & 36.6 & 55.6 & C & 73 & 1.50 & 3.7 & 49.1 & 7.6 \\
\hline 20 & $0-30$ & 7.8 & 1.03 & 18.5 & 37.6 & 43.9 & C & 68 & 0.73 & 3.4 & 32.9 & 10.4 \\
\hline 21 & $0-30$ & 8.1 & 0.67 & 6.4 & 36.8 & 56.8 & C & 77 & 2.38 & 2.6 & 49.1 & 5.3 \\
\hline 22 & $0-30$ & 7.8 & 1.87 & 13.2 & 34.5 & 53.3 & C & 76 & 1.42 & 7.4 & 44.9 & 16.5 \\
\hline 23 & $0-30$ & 8.3 & 2.92 & 9.5 & 33.8 & 56.7 & C & 78 & 2.32 & 6.4 & 44.9 & 14.3 \\
\hline 24 & $0-30$ & 8.3 & 3.31 & 24.5 & 33.8 & 41.7 & C & 67 & 2.28 & 5.0 & 34.8 & 14.5 \\
\hline 25 & $0-30$ & 8.1 & 2.06 & 14.7 & 32.7 & 52.6 & C & 74 & 2.33 & 2.2 & 44.7 & 4.9 \\
\hline 26 & $0-30$ & 8.6 & 2.24 & 12.6 & 34.5 & 52.9 & C & 74 & 3.50 & 3.5 & 43.2 & 8.2 \\
\hline 27 & $0-30$ & 7.7 & 1.68 & 4.4 & 36.9 & 58.7 & C & 79 & 1.06 & 3.9 & 45.8 & 8.6 \\
\hline 28 & $0-30$ & 7.8 & 1.57 & 12.5 & 34.7 & 42.8 & C & 68 & 0.98 & 3.1 & 32.7 & 9.5 \\
\hline 29 & $0-30$ & 8.0 & 1.52 & 4.5 & 36.9 & 58.6 & C & 79 & 1.51 & 5.3 & 51.6 & 10.3 \\
\hline
\end{tabular}

$\mathrm{CL}=$ Clay Loam, SiC = Silty Clay and C = Clay $\quad{ }^{* \star} \mathrm{FC}=$ Estimated Field Capacity 
Table (4): Cont.

\begin{tabular}{|c|c|c|c|c|c|c|c|c|c|c|c|c|}
\hline $\begin{array}{l}\text { Min. } \\
\text { No }\end{array}$ & Depth & $\mathrm{pH}$ & $\begin{array}{c}\text { ECe } \\
\text { dSm-1 }\end{array}$ & $\begin{array}{c}\text { Sand } \\
\%\end{array}$ & $\begin{array}{l}\text { Silt } \\
\%\end{array}$ & $\begin{array}{c}\text { Clay } \\
\%\end{array}$ & 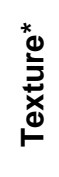 & $\begin{array}{c}\mathrm{FC}_{\%}^{\star *} \\
\%\end{array}$ & OM \% & $\begin{array}{c}\text { Ex } \\
\mathrm{Na} \\
\text { meq } \\
100 \mathbf{g}^{-1}\end{array}$ & $\begin{array}{c}\text { CEC } \\
\text { meq } \\
\mathbf{1 0 0 g}^{-1}\end{array}$ & ESP \\
\hline 30 & $0-30$ & 7.7 & 1.20 & 4.4 & 34.7 & 60.9 & C & 80 & 2.27 & 6.4 & 52.1 & 12.4 \\
\hline 31 & $0-30$ & 7.5 & 5.36 & 12.6 & 33.7 & 53.7 & C & 77 & 1.20 & 11.7 & 51.8 & 22.7 \\
\hline 32 & $0-30$ & 7.5 & 1.56 & 19.6 & 33.8 & 42.6 & C & 67 & 1.51 & 3.7 & 37.1 & 9.9 \\
\hline 33 & $0-30$ & 7.6 & 1.11 & 11.5 & 33.9 & 54.6 & C & 75 & 1.77 & 4.8 & 48.1 & 9.9 \\
\hline 34 & $0-30$ & 7.7 & 1.01 & 25.5 & 33.7 & 41.8 & C & 66 & 1.46 & 2.0 & 34.9 & 5.6 \\
\hline 35 & $0-30$ & 8.5 & 5.01 & 5.7 & 34.7 & 59.6 & C & 80 & 1.31 & 3.2 & 45.9 & 7.1 \\
\hline 36 & $0-30$ & 8.4 & 4.72 & 12.9 & 33.6 & 53.5 & C & 74 & 2.72 & 4.3 & 44.6 & 9.7 \\
\hline 37 & $0-30$ & 7.9 & 0.89 & 4.9 & 37.7 & 50.8 & C & 73.5 & 1.68 & 2.7 & 45.7 & 5.9 \\
\hline 38 & $0-30$ & 8.5 & 0.99 & 23.5 & 33.7 & 42.8 & C & 67 & 1.85 & 2.2 & 32.5 & 6.9 \\
\hline 39 & $0-30$ & 7.5 & 1.56 & 19.6 & 33.8 & 42.6 & C & 68 & 1.51 & 3.3 & 33.4 & 9.9 \\
\hline 40 & $0-30$ & 7.5 & 6.45 & 4.4 & 36.9 & 58.7 & C & 79 & 0.87 & 13.8 & 51.1 & 27.0 \\
\hline 41 & $0-30$ & 7.8 & 0.79 & 4.6 & 34.9 & 60.2 & C & 80 & 1.44 & 4.0 & 51.5 & 7.8 \\
\hline 42 & $0-30$ & 7.8 & 0.62 & 4.7 & 34.8 & 60.5 & C & 80 & 0.76 & 2.9 & 52.3 & 5.5 \\
\hline 43 & $0-30$ & 8.0 & 2.38 & 8.9 & 36.8 & 54.3 & C & 74.5 & 0.66 & 8.7 & 47.9 & 18.2 \\
\hline 44 & 0-30 & 8.2 & 0.64 & 19.5 & 36.8 & 43.7 & C & 69 & 1.05 & 1.6 & 32.5 & 4.9 \\
\hline 45 & $0-30$ & 8.03 & 3.42 & 10.8 & 34.5 & 54.7 & C & 75 & 1.76 & 2.1 & 45.4 & 4.5 \\
\hline 46 & $0-30$ & 8.3 & 1.78 & 11.4 & 33.7 & 54.9 & C & 75 & 1.73 & 2.2 & 45.1 & 4.8 \\
\hline 47 & $0-30$ & 7.8 & 0.88 & 8.5 & 36.8 & 54.3 & C & 74 & 1.85 & 3.3 & 48.1 & 6.9 \\
\hline 48 & $0-30$ & 7.9 & 1.54 & 23.2 & 33.9 & 42.9 & C & 68 & 1.71 & 2.5 & 32.4 & 7.8 \\
\hline 49 & $0-30$ & 7.3 & 6.45 & 10.3 & 33.9 & 55.8 & C & 76 & 1.41 & 11.1 & 47.9 & 23.3 \\
\hline 50 & $0-30$ & 7.5 & 4.39 & 23.8 & 34.8 & 41.4 & C & 66 & 0.87 & 0.5 & 33.7 & 1.6 \\
\hline 51 & $0-30$ & 7.6 & 2.30 & 3.9 & 35.7 & 60.4 & C & 80 & 1.13 & 2.2 & 52.1 & 4.2 \\
\hline 52 & $0-30$ & 7.5 & 1.10 & 4.8 & 34.7 & 60.5 & C & 80 & 2.64 & 2.6 & 51.5 & 5.1 \\
\hline 53 & $0-30$ & 7.6 & 1.18 & 12.5 & 12.5 & 53.7 & C & 74 & 1.82 & 7.5 & 47.7 & 15.7 \\
\hline 54 & $0-30$ & 8.0 & 1.45 & 25.4 & 33.8 & 41.8 & C & 66 & 2.48 & 4.9 & 33.5 & 14.6 \\
\hline 55 & $0-30$ & 7.8 & 0.89 & 11.6 & 33.6 & 54.8 & C & 75 & 2.95 & 3.3 & 47.7 & 7.0 \\
\hline 56 & $0-30$ & 7.8 & 1.33 & 23.5 & 34.7 & 51.8 & C & 75 & 1.07 & 5.8 & 46.9 & 12.4 \\
\hline
\end{tabular}


Table (5): Soil salinity classes in the studied area.

\begin{tabular}{|c|c|c|c|}
\hline Salinity classes $^{*}$ & $\begin{array}{c}\text { ECe } \\
\mathrm{dS} \mathrm{m}^{-1}\end{array}$ & Area in Feddan & $\%$ \\
\hline Slightly Saline & $0-4$ & 49187 & 61.20 \\
\hline Moderately Saline & $4-8$ & 4680 & 5.82 \\
\hline Highly Saline & $8-16$ & 749 & 0.93 \\
\hline Fishpond & & 24096 & 29.98 \\
\hline Urban Areas & & 1665 & 2.07 \\
\hline Total & & 80377 & 100.00 \\
\hline
\end{tabular}

*According to Soil Survey Staff (2004)

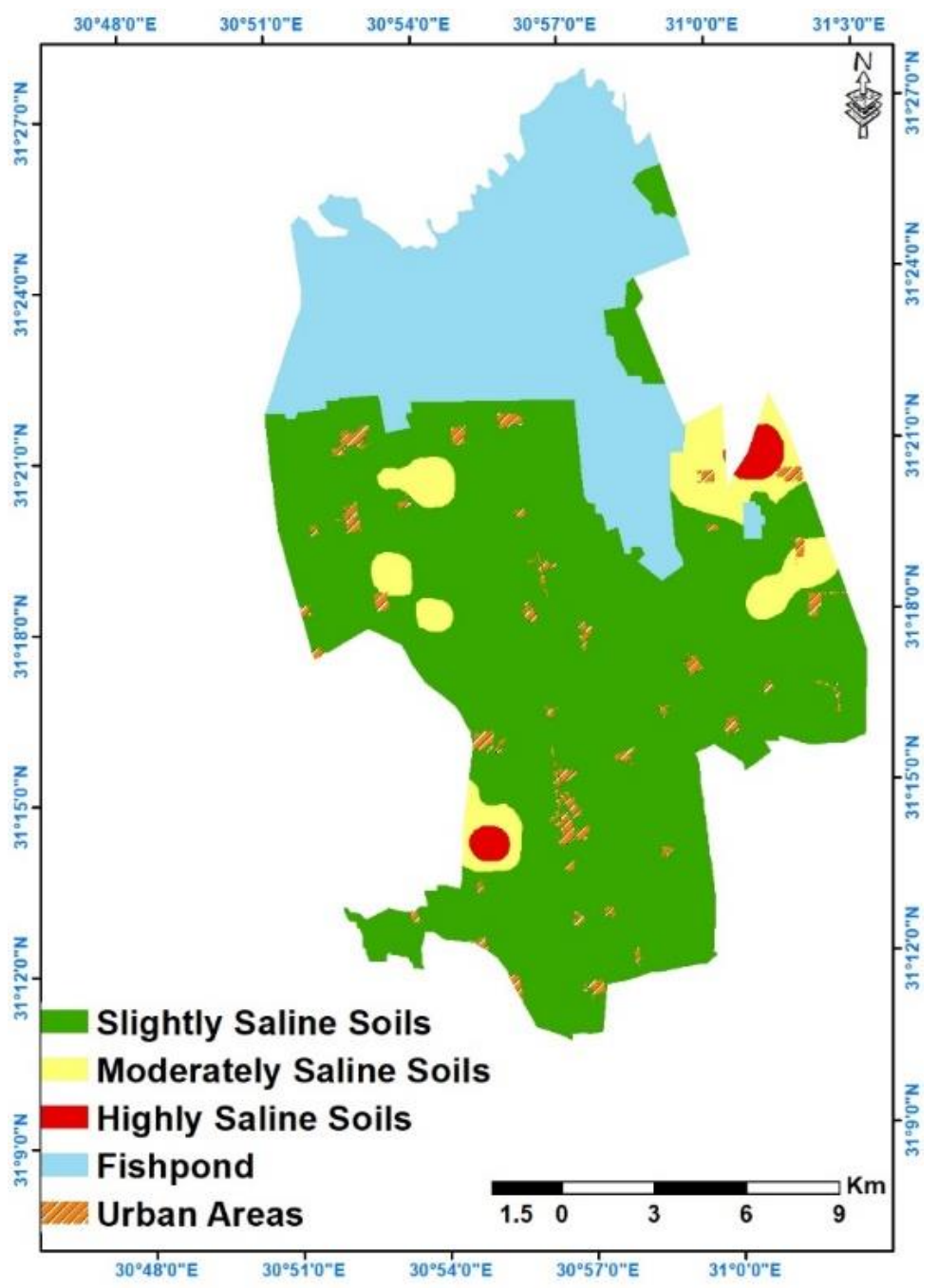

Fig. (5): Salinity map of the studied area. 
Table (6): Soil sodicity classes of the studied area*.

\begin{tabular}{|l|c|c|c|}
\hline \multicolumn{1}{|c|}{ Sodicity Classes } & ESP & Area in Feddan & $\%$ \\
\hline Non Sodic & $<15 \%$ & 48252 & 60.03 \\
\hline Sodic Soils & $>15 \%$ & 6364 & 7.92 \\
\hline Fishpond & & 24096 & 29.98 \\
\hline Urban Areas & & 1665 & 2.07 \\
\hline Total & & 80377 & 100.00 \\
\hline
\end{tabular}

${ }^{\star}$ According to Soil Survey Staff (2004)

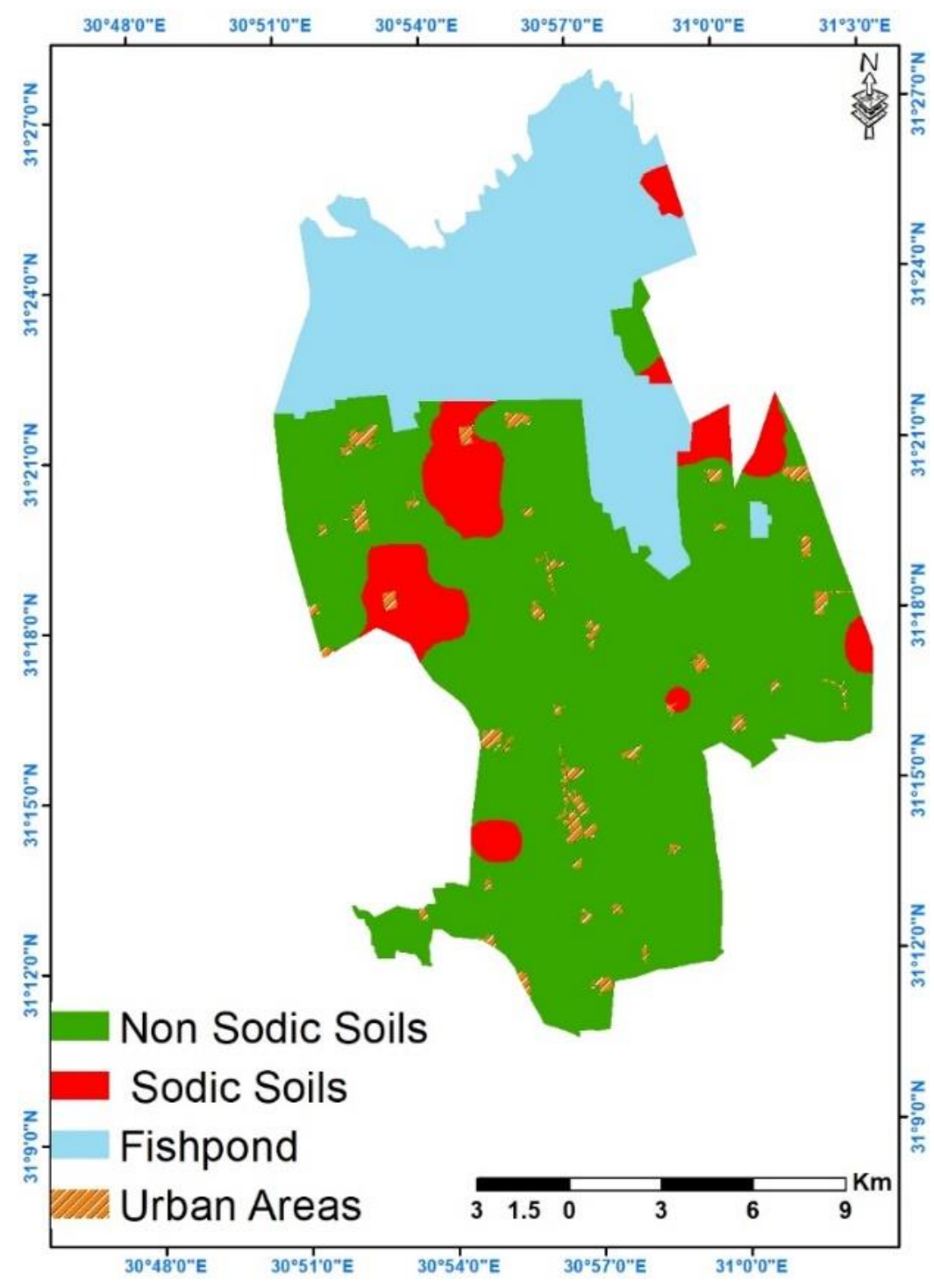

Fig. (6): Soil sodicity map of the studied area. 
Table (7): Soil OM classes of the studied area*.

\begin{tabular}{|c|c|c|c|}
\hline OM Classes & OM \% & Area in Feddan & $\%$ \\
\hline Very Low & $<0.7$ & 147 & 0.18 \\
\hline Low & $0.7-2$ & 44636 & 55.53 \\
\hline Medium & $2-3.5$ & 9832 & 12.23 \\
\hline Fishpond & & 24096 & 29.98 \\
\hline Urban Areas & & 1665 & 2.07 \\
\hline Total & & 80377 & 100.00 \\
\hline
\end{tabular}

${ }^{\star}$ According to Soil Survey Staff (2004)

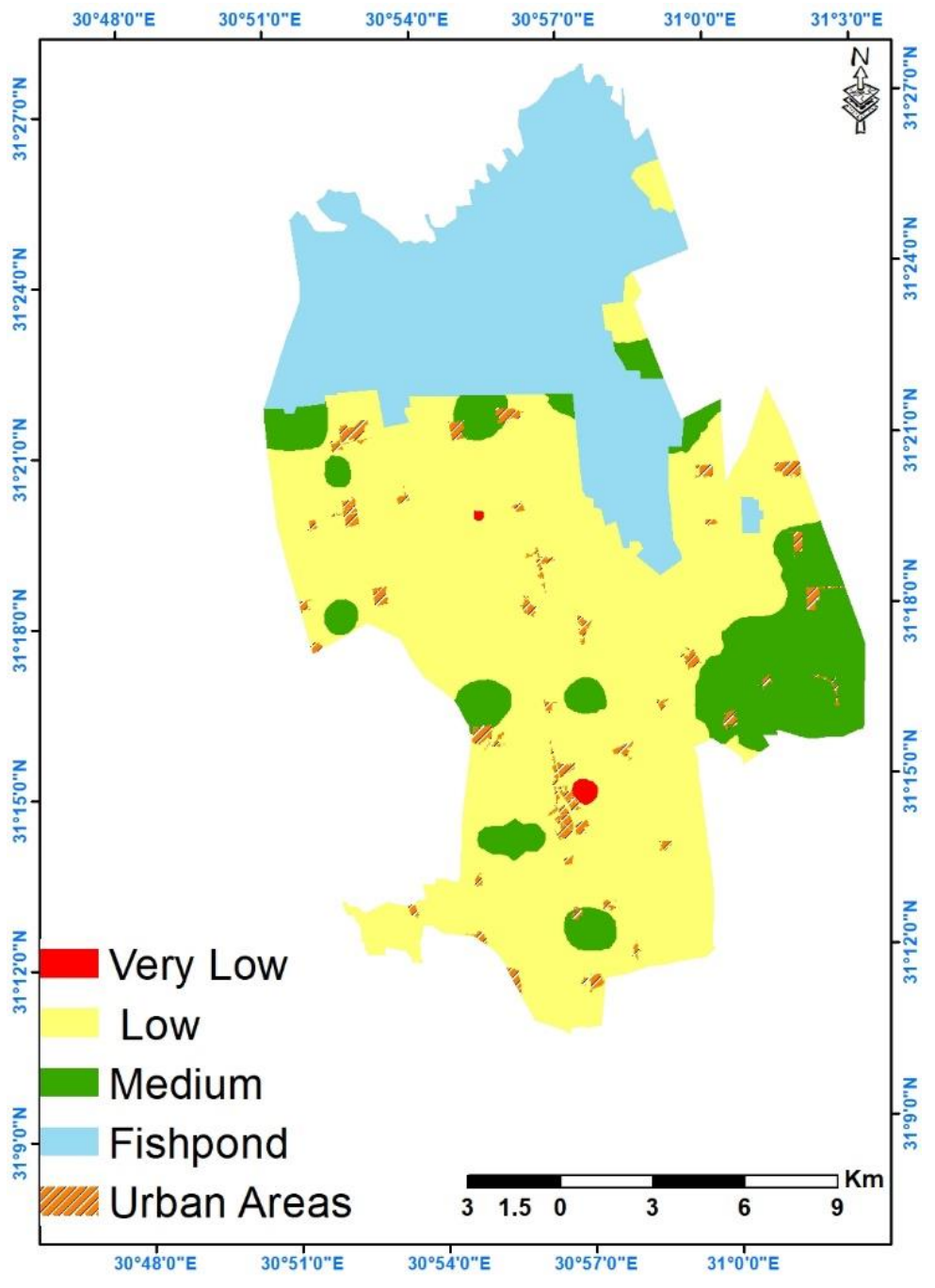

Fig. (7): Soil OM content map of the studied area. 


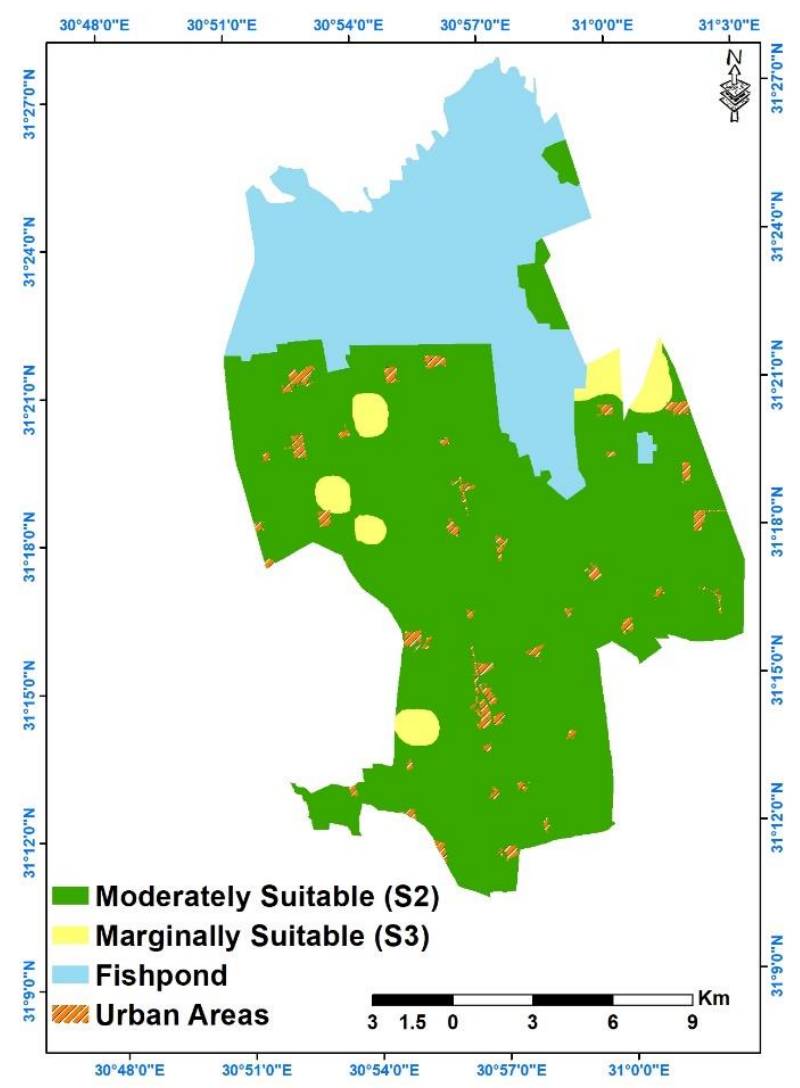

Fig. (8): Land capability map of the studied area>

Table (8): Land capability as suitability classes for agriculture*

\begin{tabular}{|l|c|c|}
\hline \multicolumn{1}{|c|}{ Capability classes } & Area in Feddan & $\%$ \\
\hline Moderately Suitable (S2) & 52000 & 64.70 \\
\hline Marginally Suitable (S3) & 2616 & 3.25 \\
\hline Fishpond & 24096 & 29.98 \\
\hline Urban Areas & 1665 & 2.07 \\
\hline Total & 80377 & 100.00 \\
\hline
\end{tabular}

${ }^{\star}$ According to Sys et al., (1991)

\section{Soil units map:}

The soils map was produced based on the spatial variability of soil salinity and soil sodicity. Six units were recognized in the studied area as shown in Fig. (9) and Table (9). Results in Fig. (9) and Table (9) reveal that the "slightly saline, non-sodic soils" is the largest soil unit occupying $56 \%$ of the total studied area. The second unit is the "slightly saline, sodic soils" occupying $5 \%$ of the area and distributes in scatter areas all over the studied area. The "moderately saline, non-sodic soils" unit covers $3.5 \%$ and distributed in small patches. The smallest unit is the "highly saline, sodic soils",that covers about $1 \%$ of the total studied area. 


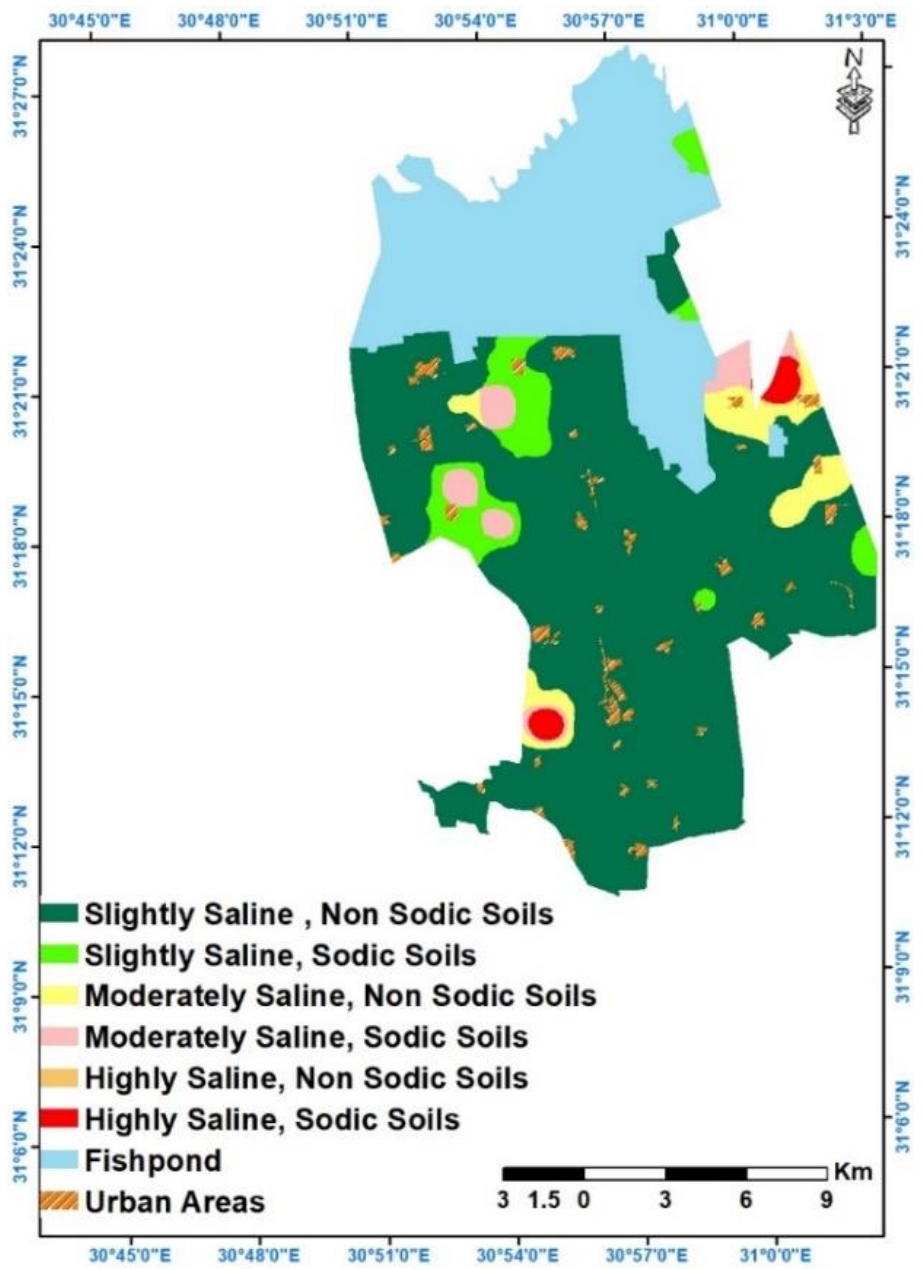

Fig. (9): Soil units map of the studied area.

Table (9): Areas of the studied soil units.

\begin{tabular}{|l|c|c|}
\hline \multicolumn{1}{|c|}{ Soil units } & $\begin{array}{c}\text { Area in } \\
\text { Feddan }\end{array}$ & $\%$ \\
\hline Slightly Saline, Non-Sodic & 45377 & 56.46 \\
\hline Slightly Saline, Sodic & 3810 & 4.74 \\
\hline Moderately Saline, Non-Sodic & 2813 & 3.50 \\
\hline Moderately Saline, Sodic & 1866 & 2.32 \\
\hline Highly Saline, Non-Sodic & 62 & 0.07 \\
\hline Highly Saline, Sodic & 687 & 0.86 \\
\hline Urban Areas & 1665 & 2.07 \\
\hline Fishpond & 24096 & 29.98 \\
\hline Total & 80377 & 100.00 \\
\hline
\end{tabular}


7. Degradation assessment in the studied area

Statistical analyses

Correlation analysis

The statistical analyses of the studied soil properties indicated that, there is a very high significant positive correlation at the 0.01 level (2-tailed) between field capacity (FC) and each of clay content \%, CEC as well as exchangeable sodium of soil samples. Whereas, there is a very high significant negative correlation at the 0.01 level (2-tailed) between FC and both of silt content $\%$, and sand content $\%$ of the soil samples (Table 10).

Table (10): Correlation between lap FC, estimated FC, and soil properties (P).

\begin{tabular}{|c|c|c|c|c|c|c|c|c|c|}
\hline Prop. & Correlations & $\mathrm{FC}^{\$}$ & eFC\$\$ & clay & silt & sand & CEC & EC & $\mathrm{Na}$ \\
\hline \multirow[t]{3}{*}{$\mathrm{FC}^{\$}$} & Pearson Correlation & 1 & $.964^{* *}$ & $.960^{* *}$ & $-.759-{ }^{* *}$ & $-.336-^{* *}$ & $.858^{* *}$ & $.218^{*}$ & $.315^{* *}$ \\
\hline & Sig. (2-tailed) & & .000 & .000 & .000 & .002 & .000 & .044 & .003 \\
\hline & $\mathbf{N}$ & 86 & 86 & 86 & 86 & 86 & 86 & 86 & 86 \\
\hline \multirow[t]{3}{*}{ eFC $\$ \$$} & Pearson Correlation & $.964^{* *}$ & 1 & $.996^{* *}$ & $-.799-* *$ & $-.381-^{* *}$ & $.897^{* *}$ & $.221^{*}$ & $.327^{* *}$ \\
\hline & Sig. (2-tailed) & .000 & & .000 & .000 & .000 & .000 & .041 & .002 \\
\hline & $\mathbf{N}$ & 86 & 86 & 86 & 86 & 86 & 86 & 86 & 86 \\
\hline \multirow[t]{3}{*}{ clay } & Pearson Correlation & $.960^{* *}$ & $.996^{\star *}$ & 1 & $-.801-^{* *}$ & $-.373-^{\star *}$ & $.928^{* *}$ & .185 & $.299^{* *}$ \\
\hline & Sig. (2-tailed) & .000 & .000 & & .000 & .000 & .000 & .089 & .005 \\
\hline & $\mathbf{N}$ & 86 & 86 & 86 & 86 & 86 & 86 & 86 & 86 \\
\hline \multirow[t]{3}{*}{ silt } & Pearson Correlation & $-.759-^{* *}$ & $-.799-* *$ & $-.801^{* *}$ & 1 & .002 & $-.775-{ }^{* *}$ & $-.105-$ & $-.293-* *$ \\
\hline & Sig. (2-tailed) & .000 & .000 & .000 & & .982 & .000 & .335 & .006 \\
\hline & $\mathbf{N}$ & 86 & 86 & 86 & 86 & 86 & 86 & 86 & 86 \\
\hline \multirow[t]{3}{*}{ sand } & Pearson Correlation & $-.336-^{* *}$ & $-.381-^{* \star}$ & $-.373-{ }^{\star \star}$ & .002 & 1 & $-.354-^{\star *}$ & $-.103-$ & -.080 \\
\hline & Sig. (2-tailed) & .002 & .000 & .000 & .982 & & .001 & .347 & .465 \\
\hline & $\mathbf{N}$ & 86 & 86 & 86 & 86 & 86 & 86 & 86 & 86 \\
\hline \multirow[t]{3}{*}{ CEC } & Pearson Correlation & $.858^{* *}$ & $.897^{* *}$ & $.928^{* *}$ & $-.775-{ }^{* *}$ & $-.354-^{* *}$ & 1 & .190 & $.364^{* *}$ \\
\hline & Sig. (2-tailed) & .000 & .000 & .000 & .000 & .001 & & .080 & .001 \\
\hline & $\mathbf{N}$ & 86 & 86 & 86 & 86 & 86 & 86 & 86 & 86 \\
\hline \multirow[t]{3}{*}{ EC } & Pearson Correlation & $.218^{*}$ & $.221^{*}$ & .185 & $-.105-$ & $-.103-$ & .190 & 1 & $.647^{* *}$ \\
\hline & Sig. (2-tailed) & .044 & .041 & .089 & .335 & .347 & .080 & & .000 \\
\hline & $\mathbf{N}$ & 86 & 86 & 86 & 86 & 86 & 86 & 86 & 86 \\
\hline \multirow[t]{3}{*}{$\mathrm{Na}$} & Pearson Correlation & $.315^{\star \star}$ & $.327^{\star *}$ & $.299^{\star *}$ & $-.2933^{* *}$ & $-.080-$ & $.364^{\star \star}$ & $.647^{\star *}$ & 1 \\
\hline & Sig. (2-tailed) & .003 & $\cdot .002$ & .005 & .006 & .465 & .001 & .000 & \\
\hline & $\mathbf{N}$ & 86 & 86 & 86 & 86 & 86 & 86 & 86 & 86 \\
\hline
\end{tabular}

**. Significant at the 0.01 level (2-tailed). $\quad \mathrm{FC}^{\$}$ Lap. field capacity

*. Significant at the 0.05 level (2-tailed). $\quad$ FC $^{\$ \$}$ estimated field capacity 
The correlation between lap FC and estimated $\mathrm{FC}$ values

The results indicated that, there is a very high significant positive correlation $\left(R^{2}=0.964^{\star \star}\right)$ at the 0.01 level (2-tailed) between estimated FC and lap FC of the soil samples. Also the results illustrated in Table (10) showed a very high significant correlation at the 0.01 level (2tailed) between estimated FC with each of clay content $\%$, silt content $\%$, sand content \%, CEC, EC, and exchangeable sodium of soil samples. A multi regression analysis was applied for estimation field capacity to improve the accuracy of obtained result.

\section{Multi Regression Analysis}

The capability of SPSS software was used to analyze the soil properties and produce ANOVA and Coefficients tables that used to calculate minipits field capacity. Tables (11 and 12) show the Analysis of Variances (ANOVA) and Coefficients parameters.

\section{Degradation indices of studied area}

The surface layer is a part of the soil section that controls degradation processes and affected by human activities and agricultural practices. The analyses results of the surface layer in the studied area were used to estimate the Chemical Degradation Index (CDI) and Biological Degradation Index (BDI) to assess the soil degradation extent.

The chemical and biological degradation indices (CDI \& BDI) were estimated according to FAO (1980).

Table (11): ANOVA parameters

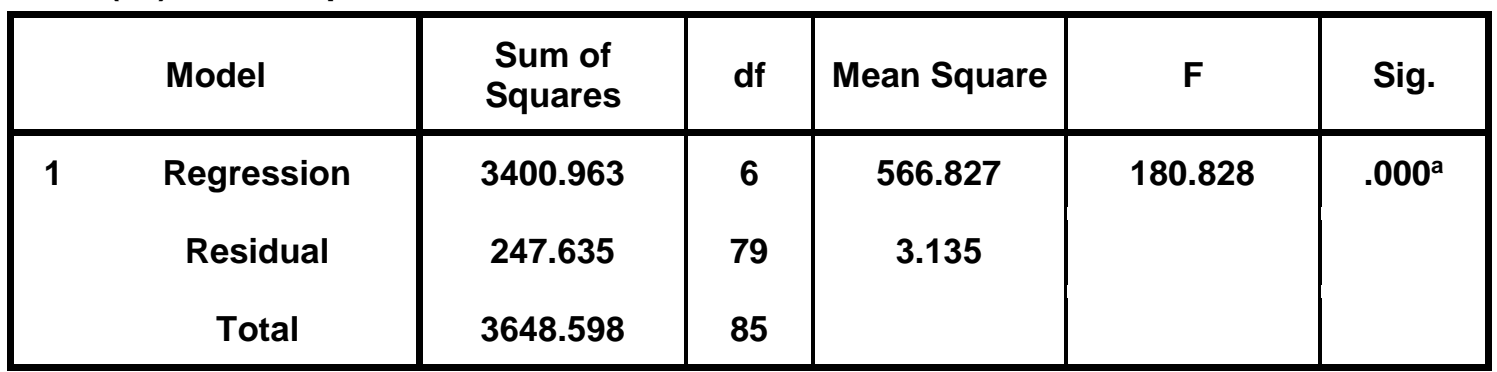

a. Predictors: (Constant), Na, sand, silt, EC, CEC, clay. b. Dependent Variable: FC

Table (12): Coefficient parameters

\begin{tabular}{|l|c|c|c|c|c|}
\hline \multirow{2}{*}{ Model } & \multicolumn{2}{c|}{$\begin{array}{c}\text { Unstandardized } \\
\text { Coefficients }\end{array}$} & $\begin{array}{c}\text { Standardized } \\
\text { Coefficients }\end{array}$ & t & Sig. \\
\cline { 2 - 5 } & B & Std. Error & Beta & & .000 \\
\hline 1 & 28.812 & 4.455 & & 13.484 & .000 \\
clay & .992 & .074 & 1.221 & .674 & .503 \\
silt & -.037 & .055 & .040 & .975 & .332 \\
sand & -.044 & .046 & .037 & $-3.019-$ & .003 \\
CEC & .213 & .071 & $-.250-$ & .525 & .601 \\
EC & .066 & .125 & .021 & 1.019 & .311 \\
\hline
\end{tabular}

a. Dependent Variable: FC 


\subsection{Chemical Degradation}

Soil data were applied to the equations 2 and 3 , that checked, modified and statistical analyzed using SPSS program. The results are presented in Table (10 to 12). The modified equations are presented as follows:

Hs $=28.812+(0.992 *$ Clay $\%)+(-$

$0.037^{\star}$ Silt\%)+(-0.044*Sand\%) (\%) ... eq.5

Salts $(\mathrm{meq} / 100 \mathrm{~g})=\left(0.066{ }^{*} \mathrm{EC}_{\mathrm{e}}{ }^{*} \mathrm{Hs}\right) / 1000$ eq. 6

A correlation analysis was performed in order to identify the most influential variables effected the CDI of the studied soils. The selected variables were soluble salts, exchangeable sodium and CEC). These variables were varied within a particular range, and their effects on CDI were estimated and illustrated in Figs (10 and 11). The results in Figs (10 and 11) revealed a very strong correlation $\left(R^{2}=\right.$ $0.93)$ between the exchangeable sodium $(\mathrm{Na})$ and $\mathrm{CDI}$, a weak correlation $\left(\mathrm{R}^{2}=\mathbf{0 . 3 6}\right)$ between the soluble salt content and CDI, and a very weak correlation $\left(R^{2}=0.02\right)$ between CEC and CDI. This means that any slight variations in the soluble salt content and the exchangeable sodium lead to a remarkable change in the chemical degradation degree. The high effect of $\mathrm{Na}^{+}$at $\mathrm{CDI}$ could be referred to their extreme levels which cannot be buffered by CEC. The high sodium content has a particular effect on the soil hydraulic properties and increases the rate of the salinity affected soils.

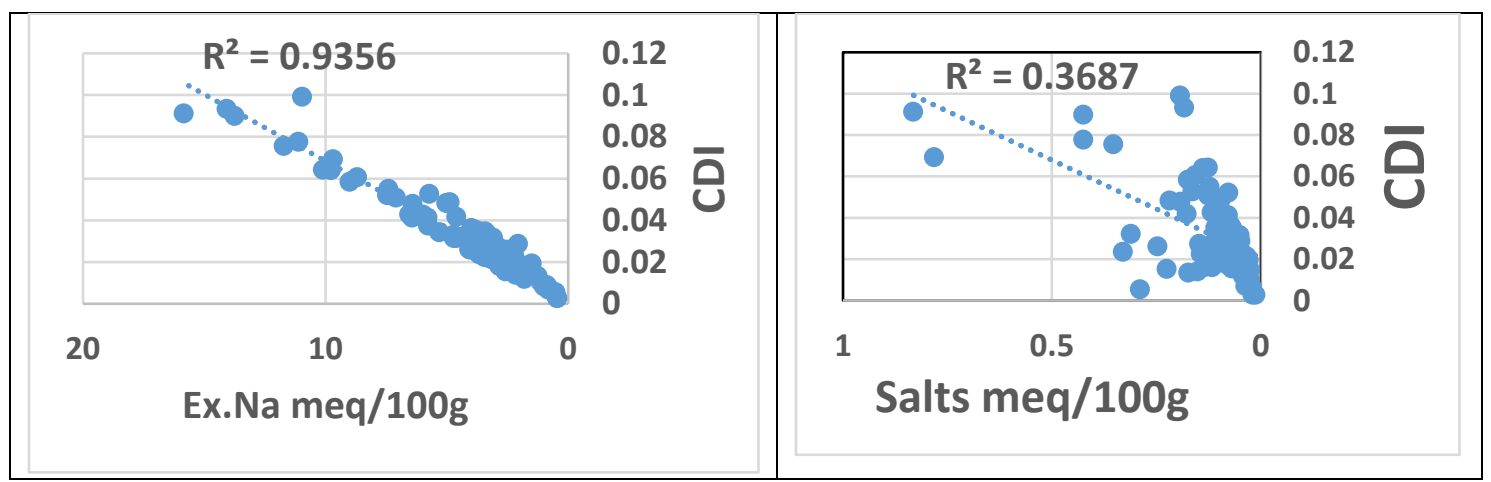

Fig. (10). Effect of exchangeable sodium and soluble salts content on CDI.

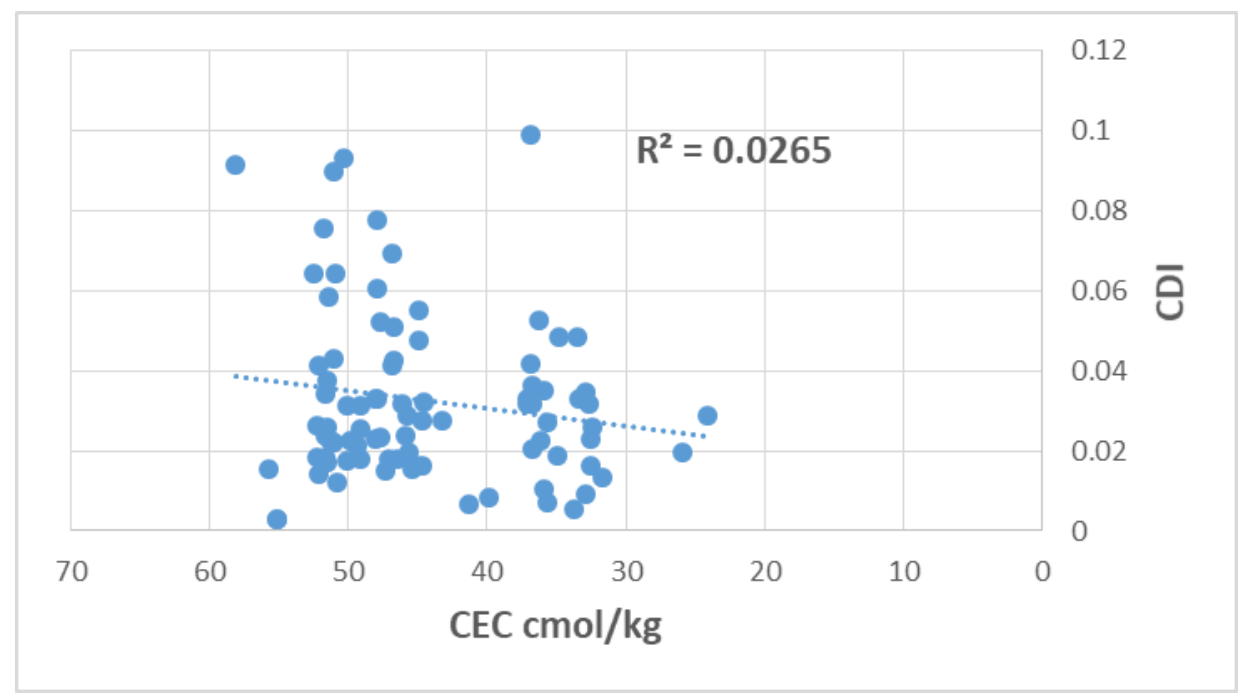


Fig. (11): Effect of cation exchange capacity on CDI.

The results of soil chemical Table (13). These results indicated that, the CDI levels vary from very low to high. The largest class of the studied area (42\%) have moderate index ( $C D I=0.02$ to 0.04 ). This area are represented by the soils of "slightly saline, non-sodic". The highly degraded index (CDI $=0.04-0.08$ ) covers about $11 \%$ of the studied area and represented by the soils of "slightly saline, moderately saline and highly saline, sodic soils".

\subsection{Biological Degradation}

Data presented in Fig. (12) and Table studied area (about $40 \%$ ) has a moderate biological degradation (BDI $=0,6$ to 1,0 ). degradation are presented in Fig. (12) and (13) indicated that, the largest class of the This soil degradation class referred to its

low organic matter content under the prevailing semiarid conditions.

\section{Drain Water quality}

Irrigation water quality plays very important role in land degradation. The quality, particularly salinity and alkalinity (SAR) are crucial for agricultural purposes. Water samples were collected from four main drain canals in the studied area. These samples were analyzed to estimate their quality and results are presented in Table (14). The results indicated that, all water samples are very highly saline $(>3 \mathrm{dS} / \mathrm{m})$ and medium sodium (C4 - S2). This drainage water is considered not suitable for agricultural irrigation. The continues use of this drainage water for irrigation could be lead to increase of soil salinity and the chemical degradation of the soil.
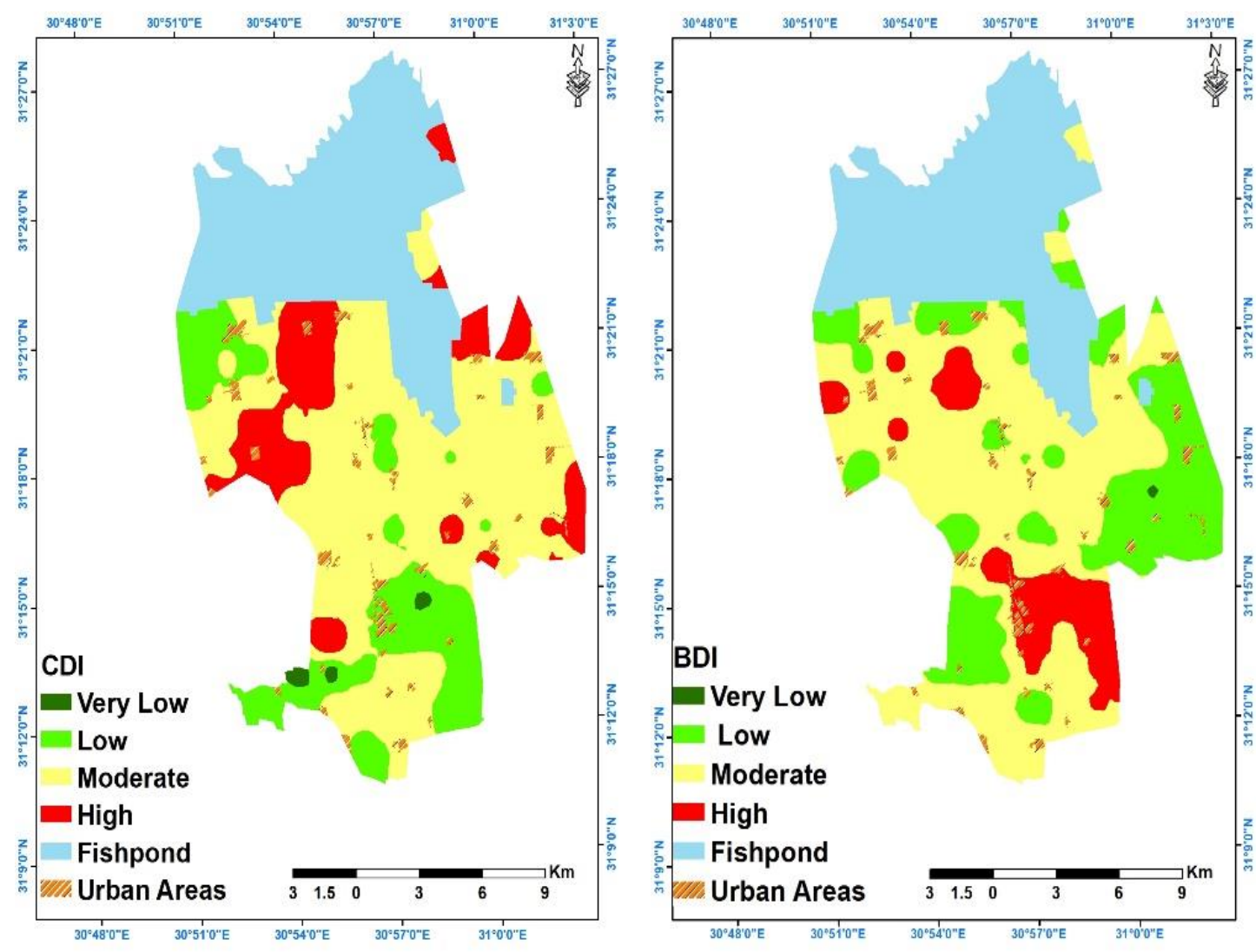
Fig. (12): Chemical (CDI) and biological (BDI) degradation status of the studied area. Table (13): Classes Areas of (CDI) and (BDI) in the studied area.

\begin{tabular}{|c|c|c|c|c|c|}
\hline CDI & $\begin{array}{c}\text { Area in } \\
\text { Feddan }\end{array}$ & $\%$ & BDI & $\begin{array}{c}\text { Area in } \\
\text { Feddan }\end{array}$ & $\%$ \\
\hline Very Low & 391 & 0.49 & Very Low & 47 & 0.06 \\
\hline Low & 11409 & 14.19 & Low & 16105 & 20.04 \\
\hline Moderate & 33849 & 42.11 & Moderate & 32191 & 40.05 \\
\hline High & 8967 & 11.16 & High & 6273 & 7.80 \\
\hline Fishpond & 24096 & 29.98 & Fishpond & 24096 & 29.98 \\
\hline Urban Areas & 1665 & 2.07 & Urban Areas & 1665 & 2.07 \\
\hline Total & 80377 & 100.00 & Total & 80377 & 100.00 \\
\hline Total & 80377 & 100.00 & Total & 80377 & 100.00 \\
\hline
\end{tabular}

Table (14): Chemical properties of drains water

\begin{tabular}{|l|c|c|c|c|c|c|c|c|c|}
\hline \multirow{2}{*}{ Drains } & \multirow{2}{*}{$\begin{array}{c}\text { EC } \\
(\mathrm{dS} / \mathrm{m})\end{array}$} & \multirow{2}{*}{ SAR } & \multicolumn{4}{|c|}{ Cations (meq/l) } & \multicolumn{3}{c|}{ Anions (meq/l) } \\
\cline { 4 - 10 } & & $\mathrm{Na}$ & $\mathrm{Ca}$ & $\mathrm{Mg}$ & $\mathrm{K}$ & $\mathrm{HCO}_{3}$ & $\mathrm{Cl}$ & $\mathrm{SO}_{4}$ \\
\hline Nashart & 7.4 & 14.48 & 51 & 15.8 & 9.0 & 0.8 & 5.5 & 35.7 & 35.4 \\
\hline Drain No. 8 & 6.5 & 15.46 & 50 & 12.5 & 8.4 & 0.7 & 5.0 & 30.1 & 36.5 \\
\hline Al Monshah & 5.6 & 13.75 & 40.1 & 11.5 & 5.5 & 0.7 & 4.5 & 28.2 & 25.1 \\
\hline Drain No. 6 & 5.5 & 12.43 & 37.4 & 11.5 & 6.6 & 0.6 & 4.5 & 26.2 & 25.4 \\
\hline
\end{tabular}

\section{Conclusion}

The using of integration of remote sensing (RS), geo-statistical analyses, and traditional statistics applied with geographic information system (GIS) is very helpful for studying the soil characteristics. These techniques are also very useful for mapping soil units, land evaluation and soil degradation. The using of statistical analyses include correlation operation, analyses of variances, and linear regression model are helpful to predicate the missing values of field capacity of the minipits soils. Therefore, the new model is valid in such soils under the Egyptian conditions.

One from the main reasons for soil degradation especially chemical degradation in Egyptian soils is the using of drainage water for irrigation due to the shortage of Nile Water. Continuous use of this drainage water leads to decrease the soil permeability caused by sodium accumulation in the exchange phase.

\section{REFERENCES}

Abdel Kawy, W., A. and R.R. Ali (2012). Assessment of soil degradation and resilience at northeast Nile Delta, Egypt: The impact on soil productivity. The Egyptian Journal of Remote Sensing and Space Sciences. 15: 1930

Barbero-Sierra, C., M. J. Marques, M. RuizPérez, R. Escadafal and W. Exbrayat (2015). How is desertification research addressed in Spain?, Land Versus Soil 
Approaches, 26 (5): 423-432, doi:10.1002/ldr.2344.

Daels, L. (1986). Remote sensing fundamentals. Gent. State Univ., Gent., ITC. J., pp.1-19.

Derici, M.R. (2002). Degradation, chemical. pp. 268-271. In R. Lal (ed.), Encyclopedia of soil science. Marcel Dekker, Inc.

De Paz, J.M., J. Sanchez and F. Visconti (2006). Combined use of GIS and environmental indicators for assessment of chemical, physical and biological soil degradation in a Spanish Mediterranean region; Journal of Environmental Management, 79: 150-162.

FAO (1980). A provisional methodology for soil degradation assessment. FAO/UNEB/Unesco.

FAO (1985). Guidelines for Land Evaluation for irrigated Agriculture. FAO Soils Bulletin No. 55, Rome, Italy.

FAO (2006). "Guidelines for soil description "fourth edition, FAO, Rome, ISBN 92-5105521-10.

Fleskens, L. and L. C. Stringer (2014). Land management and policy responses to mitigate desertification and land degradation, Land Degrad. Dev., 25: 1-4, doi: 10.1002/Idr.2272.

Hill, M. J., R. Braaten, S. M. Veitch, B. G. Lees and S. Sharma (2005). Multicriteria decision analysis in spatial decision support: the ASSESS analytic hierarchy process and the role of quantitative methods and spatially explicit analysis, Environ. Modell. Softw., 20: 955-976, doi:10.1016/j. envsoft. 2004.04.014.

Sanchez, J., R. Boluda, C. Morell, J.C. Colomer and A. Artiago (1998). Degradation index of desertification threatened soils in the Mediterranean region. Application in Castilla-La Mancha (Spain). The soil as a strategic resource: degradation processes and conservation measures. Geoforma editions, Logrono, Spain pp. 441-448.

Sanchez, J., R. Boluda, C. Morell, J.C. Colomer and A. Artiago (1999). Assessment of soil degradation within the EFEDA area. In: Balabanis, P., Peter, D., Ghazi, A., Sogas, M.T. (Eds.), Mediterranean Desertification: Research Results and Policy Implications, pp. 387-396.

Shalaby, A., R. Ali and A. Gad (2012). Land Degradation Monitoring in the Nile Delta of Egypt, using Remote Sensing and GIS. International Journal of Basic and Applied Sciences., 1 (4): 292-303.

SPSS, (2008). Statistical Package for the Social Sciences, SPSS ${ }^{\circ}$ software platform offers advanced statistical analysis, copyright polar Engineering and consulting,

http://www.winwrap.com

Stein, A. (1998). Integrating spatial statistics and remote sensing. INT. J., 19 (9): 1793-1814.

Sys, C. and W. Verheye (1978). An attempt to the evaluation of physical land characteristics for irrigation according to the FAO Framework for land evaluation. Ghent, Belgium., The Netherlands, ITC. J., pp. 66-78.

Sys, C., E. Van Ranst and J. Debavey (1991). Land Evaluation. Part I and S2, Ghent Univ., Ghent Belgium.

Tetteh, R. N. (2015). Chemical soil degradation as a result of contamination: A review. Journal of Soil Science and Environmental Management., 6 (11: 301-308.

Soil Survey Staff (2004). Soil Survey Laboratory Methods Manual, Soil Survey Investigation Report No.42: Version 4.0.

Wahab, M. A., M. A. Rasheed and R. A. Youssef (2010). Degradation Hazard Assessment of Some Soils North Nile Delta, Egypt. Journal of American Science., 6 (6):156-161. 
تقييم وإنتاج خرائط تدهور الأراضي باستخدام نظم المعلومات الجغرافية وأدلة التدهور

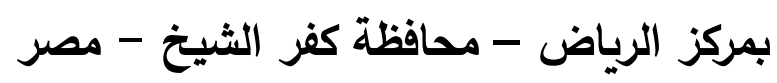

\author{
يوسف قطب الغنيمى \\ معهد بحوث الاراضى والمياه والبيئة - مركز البحوث الزراعية.
}

الملخص العبى

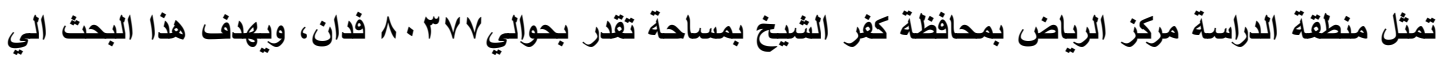

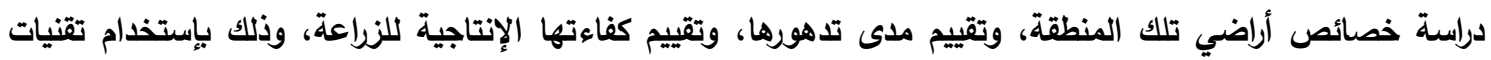

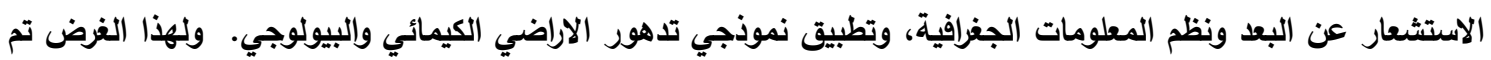

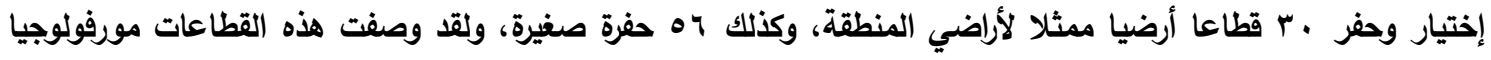

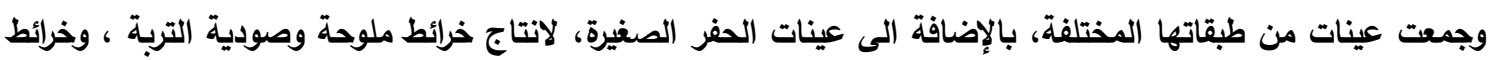

التدهور الكيمائي والبيولوجي.

درست الصفات المميزة لوحدات خريطة التربة الناتجة، وتم التعرف على الوحدات السائدة بها كالاتي:

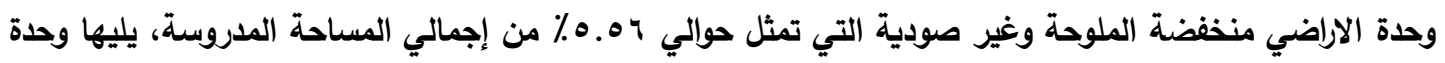

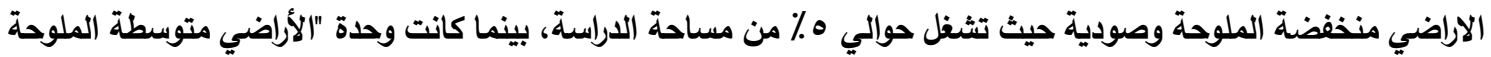

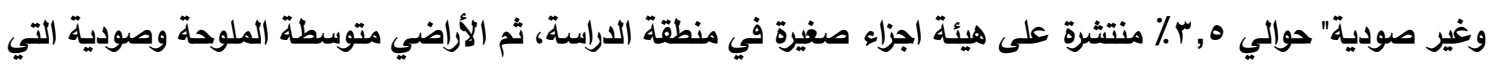

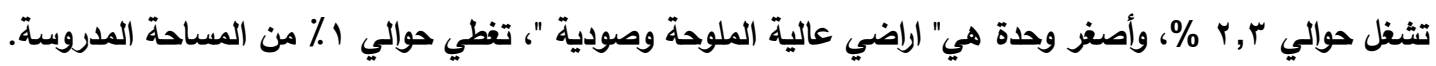

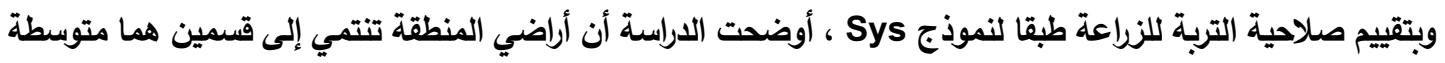

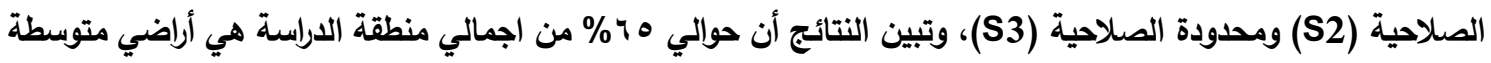

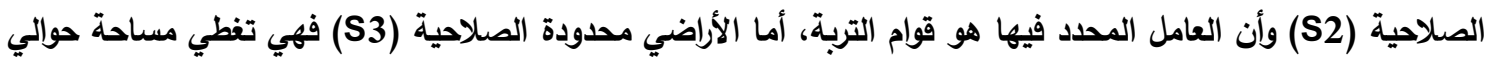

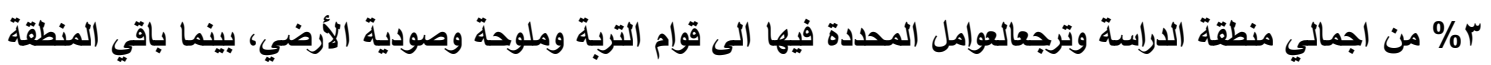
(حوالي rr\%) كانت عبارة عن مزارع سمكية ومناطق سكنية.

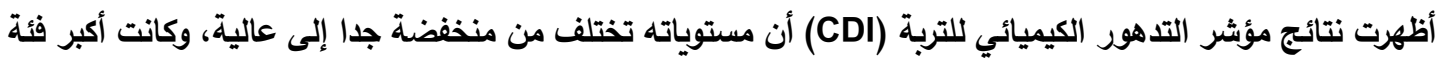

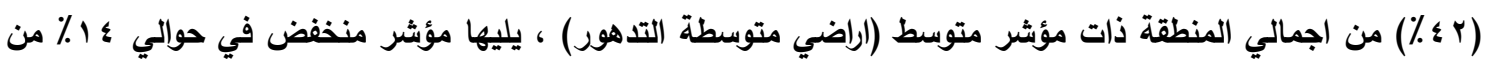

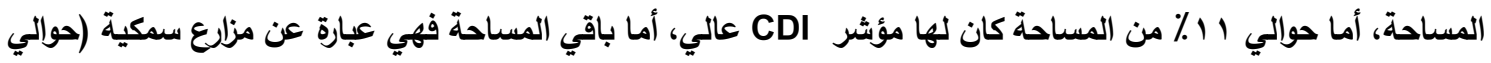
r r r r .

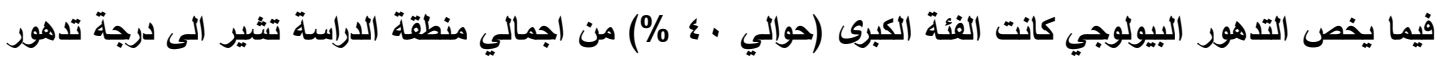
بيولوجي متوسط ، نتيجة لسرعة تحلل للمواد العضوية بسبب الظروف المناخية الفئية الجافة والثبه الجافة.

أسماء السادة المحكمين:

أ.د/ محمد سمير عراقى عميره كلية الزراعة - جامعة المنوفية

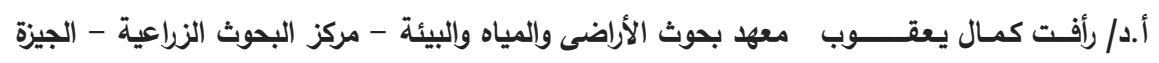


